MIT-CTP-2589

ENSLAPP-A-627/96

\title{
Kosterlitz-Thouless Phase Transitions on Discretized Random Surfaces
}

\author{
Andrei Matytsin \\ Center for Theoretical Physics \\ Laboratory for Nuclear Science \\ Massachusetts Institute of Technology \\ 77 Massachusetts Avenue \\ Cambridge, MA 02139 \\ and \\ Philippe Zaugg \\ Laboratoire de Physique Théorique ENSLAPP* \\ LAPP, Chemin de Bellevue, BP 110 \\ F-74941 Annecy-le-Vieux Cedex, France
}

\begin{abstract}
The large $N$ limit of a one-dimensional infinite chain of random matrices is investigated. It is found that in addition to the expected Kosterlitz-Thouless phase transition this model exhibits an infinite series of phase transitions at special values of the lattice spacing $\epsilon_{p q}=\sin (\pi p / 2 q)$. An unusual property of these transitions is that they are totally invisible in the double scaling limit. A method which allows us to explore the transition regions analytically and to determine certain critical exponents is developed. It is argued that phase transitions of this kind can be induced by the interaction of two-dimensional vortices with curvature defects of a fluctuating random lattice.
\end{abstract}

* URA 14-36 du CNRS, associée à l'Ecole Normale Supérieure de Lyon et à l'Université de Savoie.

e-mail: matytsin@marie.mit.edu, zaugg@lapp.in2p3.fr 


\section{Introduction}

After a period of considerable progress in the study of two-dimensional quantum gravity and noncritical strings [1] several fundamental problems in the field remained open.

The most prominent among them is, perhaps, the problem of the $c=1$ barrier [2] [3]. Indeed, string theories with the central charge of the matter $c<1$ are by now rather well understood. On the contrary, very little is known about $c>1$ strings, but their physical properties are probably quite different [4].

The problematic nature of $c>1$ theories can be partly attributed to the presence of a tachyon in their spectra. However, this is not the main obstacle in studying them. Indeed, in the random matrix model approach to string theory the cause of the difficulties appears to be more technical [3]. In fact, it is perfectly possible to write down a matrix model which in the large $N$ limit reproduces the genus expansion of a $D$-dimensional noncritical string with $D>1$. What is lacking are the analytic tools necessary to explore such models.

The reason is that, compared to $c<1$ models, string theories with $c$ larger than one have significantly more degrees of freedom. In the continuum formulation these additional degrees of freedom correspond to the transverse modes of the string, absent for $c<1$. The appearance of new modes is especially clear in the matrix model language. The dynamical variables of matrix models are Hermitian $N \times N$ matrices, each characterized by $N^{2}$ independent matrix elements. If we diagonalize a Hermitian matrix, representing it as $M=U \lambda U^{\dagger}$, these $N^{2}$ degrees of freedom can be split into $N$ eigenvalues forming the diagonal matrix $\lambda$ and $N^{2}-N$ "angular variables" encoded in the unitary matrix $U$. In $c<1$ matrix models the angular degrees of freedom decouple (or can be easily integrated out), leaving us with a theory of $N$ eigenvalues which contains all the information about the original string theory. For matrix models of $D>1$ strings this no longer occurs. As a result, in more than one dimension the dynamics of the theory depends on all of $N^{2} \gg N$ variables.

Mathematically, when the angular variables do not decouple the partition function of a matrix model involves a nontrivial integral over unitary matrices $U$. Explicit evaluation of such integrals would be useful for applications not only in matrix models but also in lattice gauge theories. In fact, the large $N$ limit of QCD can be interpreted, through the Eguchi-Kawai reduction, as a certain integral over four unitary matrices [5]. Thus even the large $N$ QCD can be viewed as a very complicated multimatrix model, where the unitary degrees of freedom play a crucial role.

In this paper we shall explore the effects of such degrees of freedom in the simplest model where these effects are nontrivial. The theory to be considered is a one-dimensional 
infinite chain of Hermitian matrices $M_{n}$ defined by the partition function 1

$$
\mathcal{Z}=\int \prod_{n=-\infty}^{+\infty} d M_{n} \exp \left\{-N \operatorname{Tr} \sum_{n=-\infty}^{+\infty}\left[\frac{\left(M_{n+1}-M_{n}\right)^{2}}{2 \epsilon}+\epsilon V\left(M_{n}\right)\right]\right\}
$$

This model is, in fact, quite interesting by itself [6] [7] [8]. It represents a one-dimensional string theory whose target space, instead of being continuous, consists of an infinite number of equidistant discrete points. Furthermore, as we shall discuss below, the large $N$ limit of this model is related by a duality transformation to the two-dimensional $O(2)$ nonlinear sigma model coupled to quantum gravity or, equivalently, to one-dimensional bosonic string theory compactified on a circle of radius $R=1 / \epsilon$.

The multimatrix chain exhibits a phase transition induced precisely by the unitary degrees of freedom discussed above [7] [9]. Indeed, in the limit of infinitely small lattice spacing $\epsilon \rightarrow 0$ our model describes the one-dimensional string theory with a continuous target space. Such a theory has $c=1$. On the other hand, when the lattice spacing gets very large the interaction of neighboring matrices $\operatorname{Tr}\left(M_{n}-M_{n+1}\right)^{2} / 2 \epsilon$ becomes negligible compared to $\epsilon \operatorname{Tr} V(M)$. Consequently, at $\epsilon=\infty$ the multimatrix chain decouples into a product of infinitely many identical one-matrix models. For cubic $V(M)$ these one-matrix models represent pure two-dimensional quantum gravity, a system with $c=0$. Therefore, the central charge of string theory corresponding to (1.1) changes from one to zero as $\epsilon$ is increased. In fact, there is evidence that this change occurs sharply at a certain value of the lattice spacing $\epsilon=\epsilon_{\mathrm{cr}}$ where the model undergoes a phase transition.

Of course, being one-dimensional the matrix chain is substantially simpler than any of the prospective $c>1$ models. However, we shall see that already in this simple theory the unitary variables produce new effects not encountered at $c<1$. Surprisingly, we find that the phase transition at $\epsilon=\epsilon_{\mathrm{cr}}$ is not the only critical point of (1.1). It turns out that in addition this model has an infinity of critical points at $\epsilon=\epsilon_{\mathrm{cr}} \sin (\pi p / 2 q)$ labelled by positive integers $p$ and $q$, where certain observables, such as the eigenvalue densities of matrices $M_{n}$, develop singularities. These singularities are of universal nature (that is, they are the same for various potentials $V(M)$ within a certain class) and evidence phase transitions. It is quite unusual, however, that none of these transitions affects the double scaling limit of the theory so that (1.1) has $c=1$ for any $\epsilon<\epsilon_{\mathrm{cr}}$.

It is rather amusing that the additional critical points fill densely the interval $\left[0, \epsilon_{\mathrm{cr}}\right]$. This shows that once the unitary degrees of freedom are taken into account the system

1 In this formula $V(M)$ is a polynomial potential such as, for example, $m^{2} M^{2} / 2+g M^{3} / 3$ or $m^{2} M^{2} / 2+g M^{4} / 4$. 
can become very complex. Perhaps, it is not entirely unlikely that a similar kind of phase structure might also arise in $c>1$ matrix models.

In addition to being on the border between $c<1$ and $c>1$ theories, there is another reason why the matrix chain is interesting. It models, in the large $N$ limit, one-dimensional string theory compactified on a circle of radius $R=1 / \epsilon$. When defined on a discretized worldsheet this theory automatically contains vortices - topologically nontrivial configurations where the string winds around the target space circle as we follow the boundary of an elementary worldsheet plaquette. These vortices are suppressed thermodynamically for large $R$ (or, equivalently, small $\epsilon$ ) but become favored as $R$ is decreased. As a result, they induce a phase transition in very much the same way the vortices of the two-dimensional $O(2)$ model drive the Berezinsky-Kosterlitz-Thouless phase transition [10]. In fact, this Kosterlitz-Thouless-type transition is precisely the transition at $\epsilon=\epsilon_{\mathrm{cr}}$ which separates the $c=1$ and $c=0$ phases of our theory.

To explore the dynamics of the Kosterlitz-Thouless phase transition in string theory is a longstanding problem [7] [9] [11]. In this paper we shall present a computation which permits a systematic treatment of the transition region. In particular, we find that the eigenvalue density at the Kosterlitz-Thouless critical point has an unusual logarithmic singularity $2 \rho(x) \propto|x| / \log (1 / \lambda|x|)$. However, we also find that the inclusion of lattice fluctuations gives rise to new Kosterlitz-Thouless-type transitions which would not occur on a regular flat lattice. These are the transitions at $\epsilon=\epsilon_{\mathrm{cr}} \sin (\pi p / 2 q)$. They arise when the fluctuating random lattice develops a curvature defect somewhere around the vortex core. If such a defect introduces negative curvature, the energy of a vortex decreases. As a consequence, the effective temperature of the Kosterlitz-Thouless transition or, equivalently, the critical value of $\epsilon$ for such vortices becomes lower than $\epsilon_{\mathrm{cr}}$.

It is necessary to emphasize that these additional transitions do not modify the double scaling limit of the matrix chain. Therefore, they do not correspond to any new phase transitions in continuum one-dimensional string theory. However, they do exhibit universality properties and this makes them worthy of consideration. Indeed, there are examples of phase transitions which appear as lattice artifacts in one model but play an important role in the continuum limit of another. For instance, the Gross-Witten phase transition in the one-plaquette model [12], which represents a lattice effect, is in the same universality class with the Douglas-Kazakov phase transition [13] occuring in continuum large $N$ Yang-Mills theory on a two-dimensional sphere.

2 This should not be confused with the logarithmic singularity in the free energy of $c=1$ models where $\rho(x) \propto|x|$ without any logarithms. 
Since the analysis presented below is somewhat lengthy we shall first summarize the main steps of our computations and state the results. This shall be done in the next section. In sections 3 and 4 we shall present the details. In section 5 we check our results by studying the case of a special interaction potential $V(M)$ for which the model can be solved exactly in terms of elementary functions. Finally, the vortex interpretation of defect-induced phase transitions shall be discussed in section 6 .

\section{Large $N$ Expansion of Infinite Matrix Chain}

The free energy of the infinite one-dimensional random matrix chain represents, in the large $N$ expansion, the string perturbation series for one-dimensional string theory with a discrete target space [6] [7]. To see this consider a cubic $V(M)=m^{2} M^{2} / 2+g M^{3} / 3$ and expand (1.1) in powers of $g$. Such expansion is a sum of all Feynman diagrams with $\varphi^{3}$ vertices and propagators which can be inferred directly from (1.1),

$$
\left\langle M_{n, \alpha \beta} M_{n^{\prime}, \gamma \delta}\right\rangle=N^{-1} \delta_{\beta \gamma} \delta_{\alpha \delta} D\left(n-n^{\prime}\right)
$$

where $\alpha, \beta, \gamma$ and $\delta$ are matrix indices and the coordinate space propagator $D\left(n-n^{\prime}\right)$ is given by

$$
D\left(n-n^{\prime}\right)=\int_{-\pi}^{\pi} \frac{d p}{2 \pi} \mathrm{e}^{i p\left(n-n^{\prime}\right)} \frac{\epsilon}{\epsilon^{2} m^{2}+4 \sin ^{2}(p / 2)} .
$$

According to these rules, the contribution of any individual Feynman graph $\Gamma$ with $V$ vertices and $G$ handles has the form

$$
g^{V} N^{2-2 G} \sum_{n_{1}, \ldots, n_{V}=-\infty}^{+\infty} \prod_{\langle i j\rangle} D\left(n_{i}-n_{j}\right)
$$

As usual, the product of propagators goes over all links of the graph $\langle i j\rangle$ whereas the integers $n_{1}, \ldots, n_{V}$ refer to the vertex positions in the one-dimensional discrete coordinate space. In the string theory language these integers parametrize the embedding of the graph $\Gamma$ into the discretised target space of the string.

The weight assigned to a given graph in this theory, the product of propagators $D\left(n_{i}-\right.$ $n_{j}$ ), is not exactly equal to the discretized Polyakov string weight. Indeed, to obtain the Polyakov weight $\exp \left(-S_{P}\left[n_{i}\right]\right)$ with

$$
S_{P}\left[n_{i}\right]=\frac{1}{2} \sum_{\langle i j\rangle} \epsilon^{2}\left(n_{i}-n_{j}\right)^{2}
$$


one would have to choose the propagator $D_{P}(n)=\exp \left(-\epsilon^{2} n^{2} / 2\right)$. Such a propagator could be generated only by a nonlocal, hard-to-deal-with matrix model. Fortunately, it is possible to argue [2] [3] that the models with these two weights are in the same universality class. The reason is that both $D$ and $D_{P}$ have the same infrared behavior in momentum space, $\tilde{D}(p) \propto 1-p^{2} / \mu^{2}$. Since in one dimensional theory there are no ultraviolet divergences the replacement of $D_{P}$ by $D$ modifies only the short-distance, nonuniversal properties of the model.

Alternatively, the large $N$ limit of the infinite matrix chain (1.1) provides a description of one-dimensional bosonic string theory compactified on a circle [7]. However, in contrast with the discretized target space picture, this second interpretation is restricted to the leading order of large $N$ expansion. That is to say, our matrix model reproduces correctly only the first term of the genus expansion for the compactified string. To explain why let us perform a duality transformation on the Feynman amplitude (2.3). As a first step, insert the momentum space representation (2.2) for each $D$ in (2.3) and do the sums over $m_{i}$ using the identity

$$
\sum_{n=-\infty}^{+\infty} \mathrm{e}^{i p n}=2 \pi \sum_{l=-\infty}^{+\infty} \delta(p+2 \pi l)
$$

The result is

$$
g^{V} N^{2-2 G} \sum_{l_{1}, \ldots, l_{V}=-\infty}^{+\infty} \prod_{\langle i j\rangle} \int_{-\pi}^{\pi} \frac{d p_{i j}}{2 \pi} \tilde{D}\left(p_{i j}\right) \prod_{k=1}^{V}\left[2 \pi \delta\left(p_{\text {tot }}^{k}-2 \pi l_{k}\right)\right]
$$

where $p_{i j}$ stands for the momentum flowing along the link $\langle i j\rangle, \tilde{D}(p)$ is the momentum space propagator and $p_{\text {tot }}^{k}=\sum_{j} p_{k j}$ equals the sum of all momenta entering the vertex number $k$. Note that the vertices conserve momentum only modulo $2 \pi$.

If $\Gamma$ is topologically spherical it is easy to interpret (2.5) in terms of compactified string theory. To this effect we replace the link momenta $p_{i j}$ in (2.5) by the string variables $X_{a}$, to be defined shortly, which will be associated with the vertices of the dual graph $\Gamma^{*}$. Since we consider a compactified string the variable $X$ must live on a circle, so that $X$ and $X+2 \pi R n$ for any $n \in \mathbb{Z}$ are all identified.

To construct $X_{a}$, for each link $\langle i j\rangle \in \Gamma$ we find a unique link $\langle a b\rangle$ of $\Gamma^{*}$ which crosses $\langle i j\rangle$ and impose the condition

$$
X_{a}-X_{b}=R p_{i j}
$$

If we fix the value of $X$ at any one vertex (say, set $X_{1}=0$ ) then equations (2.6) define $X_{a}$ as functions of $p_{i j}$ at all other vertices. In terms of these new variables (2.5) takes the form

$$
g^{V} N^{2} \prod_{a=2}^{V^{*}} \int_{-\pi R}^{\pi R} \frac{d X_{a}}{2 \pi R} \prod_{\langle b c\rangle} \tilde{D}\left(\frac{X_{b}-X_{c}}{R}\right)
$$




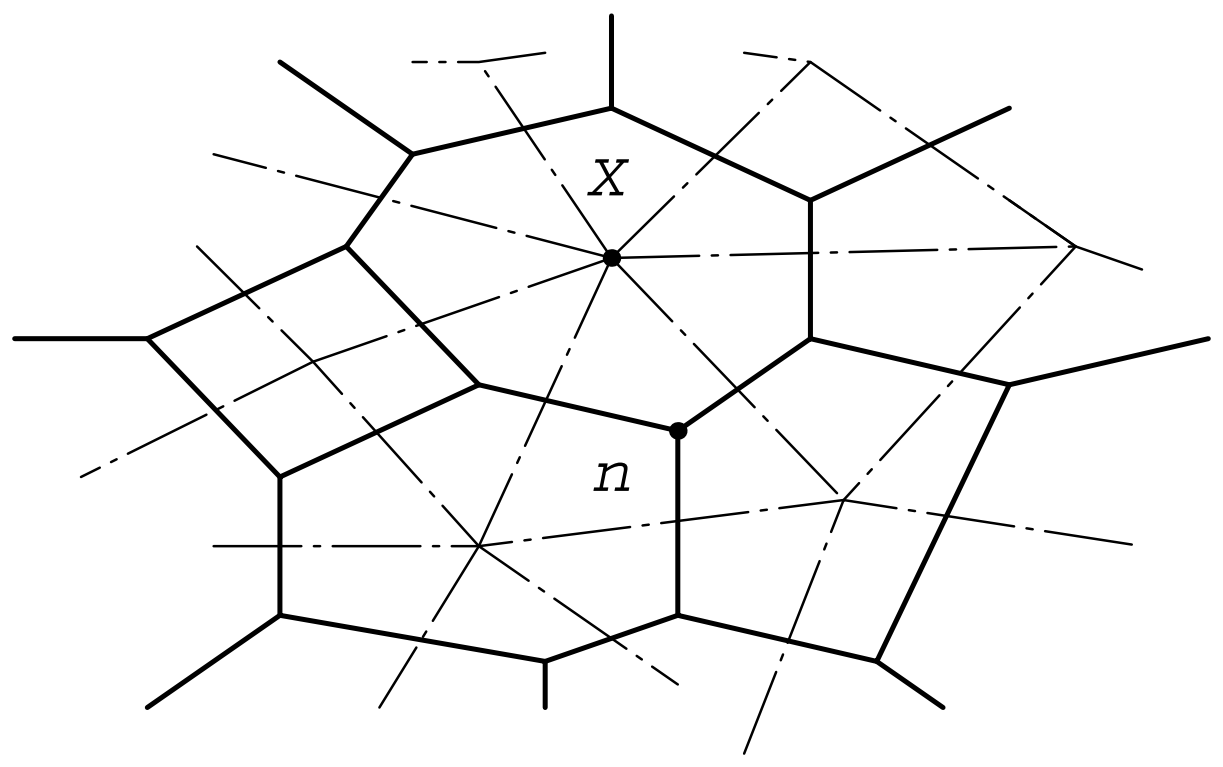

Fig. 1: A typical Feynman graph $\Gamma$ generated by a matrix model (solid lines) and its dual $\Gamma^{*}$ (dashed lines). The discrete target space coordinate $n$ is associated with the vertices of $\Gamma$ while the compactified matter field $X$ corresponds to the vertices of $\Gamma^{*}$.

which looks very similar to (2.3) except that the target space is now a continuous circle of radius $R=1 / \epsilon$.

The equivalence we just described does not hold on higher genus graphs where in general (2.6) does not have a solution. However, for our purposes this does not matter. Indeed, below we shall restrict our attention to the large $N$ limit of the chain model where only the spherical graphs survive and both interpretations apply.

To explore the large $N$ limit of the multimatrix chain we shall first integrate out the unitary degrees of freedom in (1.1) and then use the saddle point method. It will be convenient to rescale all matrices $M_{n}=\sqrt{\epsilon} \mathcal{M}_{n}$ so that $\epsilon$ disappears from the kinetic term in the partition function:

$$
\mathcal{Z}=\int \prod_{n=-\infty}^{\infty} d \mathcal{M}_{n} \exp \left\{N \operatorname{tr} \sum_{n=-\infty}^{\infty}\left[\mathcal{M}_{n} \mathcal{M}_{n+1}-U\left(\mathcal{M}_{n}\right)\right]\right\}
$$

where the potential $U(\mathcal{M})$ is related to $V(M)$,

$$
U(\mathcal{M})=\mathcal{M}^{2}+\epsilon V(\sqrt{\epsilon} \mathcal{M})
$$

As usual [14], we shall diagonalize each matrix, $\mathcal{M}_{n}=U_{n} \Lambda_{n} U_{n}^{\dagger}$ with diagonal $\Lambda_{n}=$ $\operatorname{diag}\left(\lambda_{1, n} \ldots \lambda_{N, n}\right)$ and express the Hermitian matrix measure $d \mathcal{M}_{n}$ in terms of $U$ 's and lambdas,

$$
d \mathcal{M}_{n}=\Delta^{2}\left(\lambda_{n}\right) d \lambda_{1, n} \ldots d \lambda_{N, n} d U_{n}
$$


where $\Delta\left(\lambda_{n}\right)$ is the Van der Monde determinant of eigenvalues $\lambda_{i, n}$ and $d U_{n}$ refers to the Haar measure on the unitary group $S U(N)$. Furthermore, it is useful to introduce the matrices $V_{n}=U_{n+1}^{\dagger} U_{n}$.

Given this notation the partition function of the infinite matrix chain can be written in the form

$$
\mathcal{Z}=\int \prod_{n \in \mathbb{Z}} \Delta^{2}\left(\lambda_{n}\right) d \lambda_{1, n} \ldots d \lambda_{N, n} d U_{n} \exp \left\{N \operatorname{tr} \sum_{n=-\infty}^{\infty}\left[V_{n} \Lambda_{n} V_{n}^{\dagger} \Lambda_{n+1}-U\left(\Lambda_{n}\right)\right]\right\}
$$

The matrices $V_{n}$ represent the angular degrees of freedom in our model. Fortunately, since the one-dimensional lattice does not have closed loops, all $V_{n}$ are mutually independent and we can easily integrate them out. To do this one simply changes variables from $\left\{U_{n}\right\}$ to $\left\{V_{n}\right\}$ with the result

$$
\mathcal{Z}=\int \prod_{n \in \mathbb{Z}} \Delta^{2}\left(\lambda_{n}\right) d \lambda_{1, n} \ldots d \lambda_{N, n} d V_{n} \exp \left\{N \operatorname{tr} \sum_{n=-\infty}^{\infty}\left[V_{n} \Lambda_{n} V_{n}^{\dagger} \Lambda_{n+1}-U\left(\Lambda_{n}\right)\right]\right\}
$$

Note that no Jacobian arises when we pass from $U$ to $V$.

To integrate out $V$ 's one must first compute the following unitary group integral

$$
\int d V \exp \left[N \operatorname{tr}\left(V \Lambda_{n} V^{\dagger} \Lambda_{n+1}\right)\right]=\exp \left[N^{2} F\left(\Lambda_{n}, \Lambda_{n+1}\right)\right]
$$

Then the resulting integral over the eigenvalues

$$
\mathcal{Z}=\int \prod_{n \in \mathbb{Z}} \Delta^{2}\left(\lambda_{n}\right) d \lambda_{1, n} \ldots d \lambda_{N, n} d V_{n} \exp \left\{N \sum_{n=-\infty}^{\infty}\left[N F\left(\Lambda_{n}, \Lambda_{n+1}\right)-\operatorname{tr} U\left(\Lambda_{n}\right)\right]\right\}
$$

can be evaluated, at $N \rightarrow \infty$, via the saddle point method. In fact, due to the translational invariance of the matrix chain the saddle point values of $\lambda_{i, n}$ are always independent of the site number $n$. The saddle point equations which determine these values are readily obtained by maximizing the integrand of (2.13):

$$
\left.2 N \frac{\partial F\left(\Lambda, \Lambda^{\prime}\right)}{\partial \lambda_{i}}\right|_{\Lambda^{\prime}=\Lambda}-\frac{\partial U\left(\lambda_{i}\right)}{\partial \lambda_{i}}+\frac{2}{N} \sum_{j \neq i} \frac{1}{\lambda_{i}-\lambda_{j}}=0
$$

The unitary integral (2.12) has been computed in the classic paper of Itzykson and Zuber [15]. Unfortunately though, their result is not easy to use directly in the saddle point equation (2.14). Indeed, the Itzykson-Zuber formula expresses $F$ through the determinant of a certain $N \times N$ matrix, a quantity which becomes quite complicated in the large $N$ limit. 
As $N$ goes to infinity it is convenient to characterize the eigenvalues composing the diagonal matrices $\Lambda=\operatorname{diag}\left(\lambda_{1}, \ldots, \lambda_{N}\right)$ in terms of the so-called eigenvalue densities $\rho(\lambda)$. By definition, $N \rho(\lambda) d \lambda$ is the number of those eigenvalues among $\lambda_{1}, \ldots, \lambda_{N}$ which fall into an infinitesimal interval $[\lambda, \lambda+d \lambda]$. To define the large $N$ limit of (2.12) we enlarge diagonal matrices $\Lambda_{n}$ and $\Lambda_{n+1}$ in such a way that their respective densities of eigenvalues converge to the well defined smooth limits $\rho_{n}(\lambda)$ and $\rho_{n+1}(\lambda)$. Note that as a consequence of the above definition any density $\rho(\lambda)$ always obeys the constraint

$$
\int_{-\infty}^{+\infty} \rho(\lambda) d \lambda=1 .
$$

We shall now describe, without a derivation, how one computes $F$ for infinitely large $N$. It turns out that the large $N$ asymptotics of the Itzykson-Zuber integral (2.12) is related to the classical mechanics of a certain one-dimensional integrable system [16]. This system is a droplet of one-dimensional compressible fluid which has the following peculiar equation of state

$$
P=-\frac{\pi^{2}}{3} \rho^{3}
$$

where both the local pressure $P$ and the fluid density $\rho$ depend, in general, on the onedimensional coordinate $x$. The motion of such a droplet is described by Euler equations

$$
\begin{aligned}
\frac{\partial \rho}{\partial t}+\frac{\partial}{\partial x}[\rho v] & =0 \\
\frac{\partial v}{\partial t}+v \frac{\partial v}{\partial x} & =-\frac{1}{\rho} \frac{\partial P}{\partial x} \\
& =\pi^{2} \rho \frac{\partial \rho}{\partial x}
\end{aligned}
$$

where $v(x)$ is the local velocity at point $x$.

To compute $F$ in equation (2.12) one seeks the solution of these equations which satisfies the boundary conditions

$$
\left\{\begin{array}{l}
\rho(x, t=0)=\rho_{n}(x) \\
\rho(x, t=1)=\rho_{n+1}(x)
\end{array}\right.
$$

where $\rho_{n}(x)$ and $\rho_{n+1}(x)$ are the eigenvalue densities for the matrices $\Lambda_{n}$ and $\Lambda_{n+1}$ respectively. If the functions $\rho(x, t)$ and $v(x, t)$ yield such a solution then

$$
\begin{aligned}
& \lim _{N \rightarrow \infty} F\left(\Lambda_{n}, \Lambda_{n+1}\right)=\frac{1}{2} \int_{0}^{1} d t \int d x \rho(x, t)\left[v^{2}(x, t)+\frac{\pi^{2}}{3} \rho^{2}(x, t)\right] \\
& +\frac{1}{2} \int \rho_{n}(x) x^{2} d x-\iint d x_{1} d x_{2} \rho_{n}\left(x_{1}\right) \rho_{n}\left(x_{2}\right) \ln \left|x_{1}-x_{2}\right| \\
& +\frac{1}{2} \int \rho_{n+1}(y) y^{2} d y-\iint d y_{1} d y_{2} \rho_{n+1}\left(y_{1}\right) \rho_{n+1}\left(y_{2}\right) \ln \left|y_{1}-y_{2}\right| .
\end{aligned}
$$


It is worth mentioning that this expression for $F$ has a very special structure. Most importantly, the double integral

$$
S=\frac{1}{2} \int_{0}^{1} d t \int d x \rho(x, t)\left[v^{2}(x, t)+\frac{\pi^{2}}{3} \rho^{2}(x, t)\right]
$$

is actually the value of the classical action for the fluid droplet evolving according to equations (2.16) and (2.17).

At this point we are ready to derive the large $N$ limit of the saddle point equation (2.14). First of all, we need to know the derivative $\partial F\left(\Lambda_{n}, \Lambda_{n+1}\right) / \partial \lambda_{i}$ with respect to a given eigenvalue $\lambda_{i}$. At large $N$ such a derivative can be easily expressed (simply by manipulating the definitions) through the functional derivative of $F\left[\rho_{n}, \rho_{n+1}\right]$ with respect to $\rho_{n}$,

$$
N \frac{\partial F\left(\Lambda_{n}, \Lambda_{n+1}\right)}{\partial \lambda_{i}}=\left.\left(\frac{\partial}{\partial x} \frac{\delta F\left[\rho_{n}, \rho_{n+1}\right]}{\delta \rho_{n}(x)}\right)\right|_{x=\lambda_{i}} .
$$

Furthermore, using (2.19) and equations of motion (2.16) it is possible to check that

$$
\frac{\partial}{\partial x} \frac{\delta S\left[\rho_{n}, \rho_{n+1}\right]}{\delta \rho_{n}(x)}=-v(x, t=0)
$$

and, therefore,

$$
\frac{\partial}{\partial x} \frac{\delta F\left[\rho_{n}, \rho_{n+1}\right]}{\delta \rho_{n}(x)}=-v(x, t=0)-x+2 f \frac{\rho(y) d y}{x-y} .
$$

These relations are very natural. Indeed, the variation of the action $S$ on any classical solution with respect to the initial coordinate of that solution (in this case $\rho_{n}(x)$ ) always equals minus the corresponding canonical momentum.

We can use $(2.20)$ and $(2.22)$ to simplify the saddle point equation (2.14). Keeping in mind that for $N \rightarrow \infty$

$$
\frac{1}{N} \sum_{j \neq i} \frac{1}{\lambda_{i}-\lambda_{j}} \rightarrow f \frac{\rho(y) d y}{\lambda_{i}-y}
$$

one easily reduces the saddle point equation to

$$
v(x, t=0)=\frac{1}{2} U^{\prime}(x)-x .
$$

The mathematical setup of the problem is therefore as follows. Given the potential $U(x)$ one is looking for the solution of Euler equations (2.16) which satisfies the boundary conditions

$$
\left\{\begin{array}{l}
\rho(x, t=0)=\rho(x, t=1) \\
v(x, t=0)=\frac{1}{2} U^{\prime}(x)-x
\end{array}\right.
$$


Once this solution is found the saddle point density $\rho(x, t=0)=\rho(x, t=1) \equiv \rho(x)$ can be used to compute $\mathcal{Z}$ or the free energy $\mathcal{F}=\log \mathcal{Z}$. Indeed, for the quartic $U(x)=$ $m^{2} x^{2} / 2+g x^{4} / 4$ it is easy to show that, as a consequence of (2.13) and (2.14)

$$
\frac{\partial \mathcal{F}}{\partial g}=\frac{N^{2}}{4} \int \rho(x) x^{4} d x
$$

which immediately yields $\mathcal{F}(g)$ if $\rho(x)$ is known.

To say the same differently, imagine a fluid droplet of spatially dependent density $\rho(x)$ (to be determined) being pushed with known spatially dependent initial velocity $v(x)=U^{\prime}(x) / 2-x$. Demand that after one unit of time the density of that droplet evolves into the same $\rho(x)$. This condition fixes $\rho(x)$, hopefully uniquely, for any given $v(x)$.

Most remarkably, Euler equations (2.16) are explicitly integrable. This becomes clear if we combine $v$ and $\rho$ into one complex-valued function $f=v+i \pi \rho$. One discovers that the two equations (2.16) are equivalent to the following complex equation on $f$

$$
\frac{\partial f}{\partial t}+f \frac{\partial f}{\partial x}=0
$$

commonly known as the Hopf equation. The Cauchy problem for the Hopf equation is easily solvable. If $f_{0}(x)=f(x, t=0)$ is the initial value of the function $f$ then the value of $f(x, t)$ can be found from the implicit equation

$$
f(x, t)=f_{0}[x-t f(x, t)]
$$

Now we can impose the boundary conditions (2.24) in a more explicit form. By manipulating the implicit solution (2.27) it is possible to show [16] that (2.24) gives rise to a functional equation on $\rho(x)$. This functional equation is most easily formulated in terms of two auxiliary functions

$$
G_{ \pm}(x)=\frac{1}{2} U^{\prime}(x) \pm i \pi \rho(x)
$$

and reads

$$
G_{+}\left[G_{-}(x)\right]=x .
$$

It provides a direct relation between the interaction potential and the large $N$ eigenvalue density of the infinite matrix chain.

Unfortunately, little is known about solutions of such functional equations [17]. Certainly, it seems difficult to solve (2.29) exactly for an arbitrary $U(x)$. This, however, is not always necessary. Indeed, for most purposes one is interested only in the universal properties of $\rho$-those independent of the particular $U(x)$ chosen. For example, if a matrix model 
undergoes a phase transition the eigenvalue density $\rho(x)$ usually develops a singularity at the transition point. Such singularities are typically of the form $\rho(x) \propto|x|^{\delta}$ (if the singular point is at $x=0$ ) characterized by a universal exponent $\delta$. In fact, $\delta$ is closely related to the string susceptibility $\gamma_{\mathrm{str}}=1-\delta$, a quantity which plays an important role in string theory.

Therefore, one might try to evaluate such exponents without finding the whole $\rho$. Luckily, equation (2.29) is well suited for just that. For concreteness and to make our problem well defined we shall concentrate on even potentials bounded from below, such as

$$
U(x)=-\frac{\mu^{2} x^{2}}{2}+\frac{g x^{4}}{4} .
$$

Any other (say, cubic) potential can be treated the same way. To set the notation let us choose $V(M)$ in the original definition (1.1) as 3

$$
V(M)=-2 M^{2}+\tilde{g} M^{4} / 4 .
$$

This, according to (2.9) means that

$$
\left\{\begin{array}{l}
\mu^{2}=2\left(2 \epsilon^{2}-1\right) \\
g=\tilde{g} \epsilon^{3}
\end{array}\right.
$$

For any given fixed $\epsilon$ the multimatrix chain can be in one of the two phases, depending on the value of $g$. For large $g$ (strong coupling) the height of the hump in the potential $V(M)$ is relatively low so that the eigenvalue density $\rho(x)$ has only a small dip around $x=0$. For very small $g$, however, the hump becomes high and the "eigenvalue droplet" splits into two disconnected pieces. In between these two phases at a certain $g=g_{\mathrm{cr}}(\epsilon)$ there is a phase transition where $\rho(x)$ just vanishes at $x \rightarrow 0$,

$$
\rho(x) \propto|x|^{\delta(\epsilon)} .
$$

The critical properties of the matrix chain encoded in $\delta(\epsilon)$ are not the same for various $\epsilon$. In fact, this model possesses a number of multicritical points - special values of epsilonwhere $\delta$ or other exponents can abruptly change values. One of the problems we shall address below is to find and investigate such multicritical points.

Before we proceed further it would be convenient to summarize our findings:

3 For a general quartic double well potential we can always rescale $M$ and $\epsilon$ in (1.1) to make the coefficient at $M^{2}$ equal -2 . The physics of the model is not affected by such rescaling. 

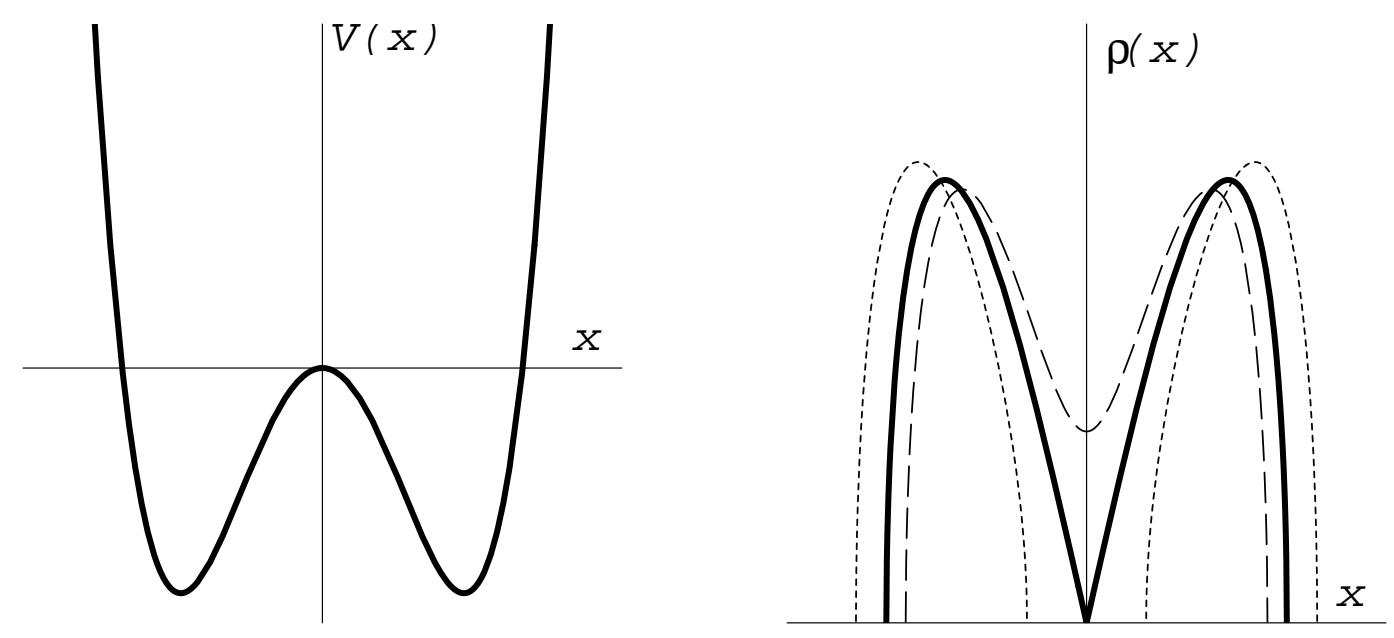

Fig. 2: A typical double-well potential $V(x)$ and the corresponding eigenvalue density $\rho(x)$ in the $c=1$ matrix model at the critical coupling $g=g_{\mathrm{cr}}$ (thick solid line), in the weak coupling phase $g<g_{\mathrm{cr}}$ (dotted line) and for strong coupling $g>g_{\text {cr }}$ (dashed line).

(a) If $0<\epsilon<1$ then $\delta(\epsilon)=1$, an old result established originally by Gross and Klebanov [7]. This value of $\delta$ is typical of $c=1$ theories. That is to say, the discrete structure of the target space does not affect the string theory so long as the target space lattice spacing is less than one.

(b) The Kosterlitz-Thouless point $\epsilon=1$ is special. It separates two regimes where the infinite matrix chain has different values of $\delta$ and describes different string theories. We find that for $\epsilon=1$ the critical eigenvalue density (that is, the density at $g=g_{\text {cr }}$ ) develops a logarithmic singularity

$$
\rho(x) \propto \frac{|x|}{\log [1 /(\lambda|x|)]} .
$$

However, the universal properties of the matrix chain at the Kosterlitz-Thouless point are not exhausted by that. As we shall prove, an infinite number of corrections to this logarithmic singularity also happen to be universal. More precisely, the critical density $\rho(x)$ can be expanded in the form

$$
\rho(x)=|x| \sum_{q=0}^{\infty} \sum_{p=1}^{q} a_{p q} l^{p} y^{-q-1}+\mathcal{O}\left(x^{2}\right)
$$


where $y \equiv \log [1 /(\lambda|x|)]$ and $l \equiv \log y$. The numbers $a_{p q}$ which arise as coefficients of this expansion are entirely independent of the interaction potential $V(M)$. The potential enters only through the nonuniversal scale $\lambda$ and through the terms of order $\mathcal{O}\left(x^{2}\right)$. We shall also see that the series in (2.35) can be summed. The result is

$$
\rho(x)=|x| \sigma(x)
$$

where $\sigma(x)$ is determined by the transcendental equation

$$
\frac{1}{\sigma}+\log \sigma=\log \left(\frac{1}{\lambda|x|}\right)
$$

Quite remarkably, nothing in this equation except for $\lambda$ depends on the matrix model potential. Therefore, equation (2.37) ought to have a continuum, string-theoretic interpretation. In the continuum language the phase transition at $\epsilon=1$ is induced by topologically nontrivial string configurations - vortices, which become strongly coupled for $\epsilon>1$. From this point of view 2.37) might contain information about vortex dynamics in the region where the "vortex gas" ceases to be dilute.

(c) Surprisingly, the linear matrix chain has other special points. To observe them it is necessary to evaluate corrections to (2.33). For a generic $\epsilon \in] 0,1[$ these corrections are of the form

$$
\rho(x)=|x|\left\{a_{1}(\epsilon)+a_{2}(\epsilon) x^{2}+a_{3}(\epsilon) x^{4}+\ldots\right\}
$$

where the coefficients $a_{k}(\epsilon)$ are fixed uniquely by Euler equations. However, if $\epsilon=$ $\sin (\pi p / 2 q) \equiv \epsilon_{p q}$ we discover that the coefficient $a_{q}(\epsilon)$ goes to infinity. At these values of $\epsilon$ the corrections to $\rho(x)$ cannot be expanded in a power series. Indeed, we shall see that at $\epsilon=\epsilon_{p q}$ the correct expansion for $\rho(x)$ contains a subleading logarithmic singularity

$$
\rho(x)=|x|\left\{a_{1}+a_{2} x^{2}+\ldots+a_{q-1} x^{2(q-2)}+a_{q} x^{2(q-1)} \log \left(\frac{1}{\lambda|x|}\right)+\ldots\right\} .
$$

These logarithmic singularities are universal. That is to say, they remain present if the quartic interaction is replaced by any other generic polynomial potential $V(M)$. It is also possible to construct a number of special, "fine-tuned" potentials for which some of the singularities get eliminated.

Mathematically, such logarithmic singularities are quite analogous to the KosterlitzThouless singularity at $\epsilon=1$. For example, if we shift $g$ away from $g_{\mathrm{cr}}(\epsilon)$ the shapes of $\rho(x)$ at $\epsilon=1$ and at $\epsilon=\sin (\pi p / 2 q)$ are given by very similar expressions. The qualitative pictures of what happens at these points seem to be related as well. Indeed, there is evidence that the singularities at $\epsilon=\epsilon_{p q}$, like the Kosterlitz-Thouless phase transition at 
$\epsilon=1$, are due to effects of vortex proliferation. Such proliferation is, of course, impossible for any $\epsilon<1$ in flat spacetime or on a regular lattice. On a random lattice the situation is different. There a pair consisting of a vortex and a negative curvature defect centered close to each other may become a favored configuration even when $\epsilon<1$. We shall see that although such pairs do not make a leading singular contribution to the eigenvalue density or the free energy of the model, they are still sufficient to cause a mild singularity in $\rho(x)$.

(d) If $\epsilon>1$ and $V(M)$ is quartic the Euler equations are consistent with $\delta(\epsilon)=2$. This is the same value of $\delta$ one observes for $\epsilon \rightarrow \infty$ when the matrix chain becomes a collection of infinitely many decoupled one-matrix models. The transition between $\delta=1$ at $\epsilon<1$ to $\delta=2$ for $\epsilon>1$ has an interesting interpretation in terms of the hydrodynamic picture. It turns out that for $\epsilon>1$ the motion of the liquid droplet prescribed by (2.16) and (2.24) results in the formation of a shock. That is, at a certain moment in time, $t_{\mathrm{sh}}=1 / 2 \epsilon^{2}$ the spatial derivative of $\rho(x, t)$ at $x=0$ becomes infinite. This phenomenon never happens for $\epsilon<1$. Moreover, the droplet picture provides a natural order parameter for the KosterlitzThouless transition. This order parameter is defined by $\zeta(\epsilon)=\rho(x=0, t=1 / 2)$ where $\rho(x, t)$ is the solution corresponding to $g=g_{\mathrm{cr}}(\epsilon)$. The quantity $\zeta(\epsilon)$ has the property

$$
\left\{\begin{array}{lll}
\zeta(\epsilon)=0 & \text { if } \quad \epsilon \leq 1 \\
\zeta(\epsilon)>0 & \text { if } \quad \epsilon>1
\end{array}\right.
$$

and will play an important role in our analysis of the $\epsilon>1$ phase.

(e) Finally, we shall study the character expansion of the infinite matrix chain. The idea is to expand the integrand of the partition function (2.11) in a certain sum over $U(N)$ characters [18]. Then within each term of this sum the unitary degrees of freedom $V_{n}$ can be integrated out with ease. At large $N$ the resulting sum over representations of $U(N)$ is dominated by a single "saddle point representation." The highest weights of this representation $\left(n_{1}, \ldots, n_{N}\right)$ are all integers of order $N$. It is conventional to characterize such highest weights by the "Young tableau density" $\rho_{l}(h)$ defined as the density of points $h_{i}=\left(n_{i}-i\right) / N+1 / 2$ in a small interval around $h$. Note that since the highest weights are ordered the Young tableau density can never be greater than one. We shall prove that the Young tableau density $\rho_{l}(h)$ is directly related to the density $\rho_{1 / 2}(x)=\rho(x, t=1 / 2)$ which arises in the hydrodynamic picture:

$$
\pi \rho_{1 / 2}\left[\sqrt{h} \cos \left(\frac{\pi \rho_{l}(h)}{2}\right)\right]=2 \sqrt{h} \sin \left(\frac{\pi \rho_{l}(h)}{2}\right) .
$$

This relation shows that there is a lot in common between the Kosterlitz-Thouless phase transition and the Douglas-Kazakov-type transitions which are observed in twodimensional QCD or in dually weighted graph models [13] [18]. In the Douglas-Kazakov 
transitions the Young tableau density is always less than one for weak coupling but develops a plateau with $\rho_{l}(h)=1$ for any $h \in\left[-h_{*}, h_{*}\right]$ at strong coupling. Exactly the same happens in the matrix chain. Indeed, since for $h=h_{*}$ the density $\rho_{l}\left(h_{*}\right)=1$, equation (2.41) predicts

$$
\zeta=\rho_{1 / 2}(0)=\frac{2}{\pi} \sqrt{h_{*}} .
$$

Equation (2.40) now implies that the plateau in $\rho_{l}$ is present only for $\epsilon>1$, in complete analogy with the Douglas-Kazakov transition. The physical pictures of the two transitions are also analogous. Indeed, both the Kosterlitz-Thouless and the Douglas-Kazakov transitions are induced by the topologically nontrivial configurations (vortices and instantons, respectively) [13] [19] which dominate in the strong coupling phases of two-dimensional $O(2)$ model and two-dimensional QCD.

A more detailed analysis of the infinite matrix chain at $\epsilon>1$ will be given separately in another report. Below we shall discuss the Kosterlitz-Thouless phase transition and the subleading logarithmic singularities which arise in the $\epsilon \leq 1$ phase of the model.

\section{Critical Behavior of Eigenvalue Densities for $\epsilon<1$}

In this section we shall explore the critical properties of the eigenvalue density for lattice spacings smaller than one.

Let us first concentrate on the case when the coupling constant $g$ is exactly equal to the critical value $g_{\mathrm{cr}}(\epsilon)$. In the language of continuum theory this would correspond to setting the renormalized cosmological constant to zero. Furthermore, since the singularity of $\rho(x)$ occurs at $x=0$ we can restrict our attention to small values of $x$.

For quartic $U(x)$ given by (2.30) and (2.32) the boundary conditions (2.24) read

$$
\begin{aligned}
v(x, t=0) \equiv v_{0}(x) & =\frac{1}{2} U^{\prime}(x)-x \\
& =-2 \epsilon^{2} x+\frac{g x^{3}}{2} .
\end{aligned}
$$

Naively, when $x$ is small we can neglect the cubic term in $v$ and consider the simplified boundary problem where the initial velocity is given by

$$
v_{0}(x)=-2 \epsilon^{2} x
$$

The solution of Euler equations subject to this boundary condition is easy to construct. We simply observe that the ansatz

$$
\left\{\begin{array}{c}
\pi \rho(x, t)=\alpha(t)|x| \\
v(x, t)=\beta(t) x
\end{array}\right.
$$


is consistent with Euler equations (2.16) provided

$$
\left\{\begin{array}{c}
\dot{\alpha}+2 \alpha \beta=0 \\
\dot{\beta}+\beta^{2}=\alpha^{2}
\end{array}\right.
$$

where the dot denotes the time derivative.

These equations can be easily solved. One introduces the complex valued function $f_{1}(t)=\beta(t)+i \alpha(t)$ which, as a consequence of (3.4) obeys

$$
\dot{f}_{1}+f_{1}^{2}=0
$$

so that

$$
f_{1}(t)=\frac{1}{t-c_{0}}
$$

with a certain complex valued constant $c_{0}$. This constant should be determined from the boundary conditions. The first condition in (2.24) amounts to $\operatorname{Im} f_{1}(0)=\operatorname{Im} f_{1}(1)$ which entails

$$
c_{0}=\frac{1}{2}+i \tau_{0}
$$

with real $\tau_{0}$. Then from the condition on the initial velocity, $\operatorname{Re} f_{1}(0)=-2 \epsilon^{2}$ one determines the constant $\tau_{0}$

$$
\tau_{0}=\frac{1}{2 \epsilon} \sqrt{1-\epsilon^{2}}
$$

and, finally, the eigenvalue density at small values of $x$

$$
\begin{aligned}
\pi \rho(x, t=0) & =\alpha(0)|x| \\
& =2 \epsilon \sqrt{1-\epsilon^{2}}|x| .
\end{aligned}
$$

The critical exponent for such $\rho$ is obviously $\delta(\epsilon)=1$. Furthermore, we see that the expression for $\rho(x)$ given by (3.8) degenerates when $\epsilon=1$. This is the first sign of the Kosterlitz-Thouless phase transition which occurs at that point.

The simple solution we just constructed exhibits several important general features. First, it is symmetric with respect to time reflection around $t=1 / 2$ :

$$
\left\{\begin{array}{l}
\rho(x, 1-t)=\rho(x, t) \\
v(x, 1-t)=-v(x, t) .
\end{array}\right.
$$

This property is in fact true for any solution of Euler equations which obeys boundary conditions (2.24). The reason is that both Euler equations and the boundary conditions are invariant with respect to the reversal of time. That is to say, if $\rho(x, t)$ and $v(x, t)$ yield 
a solution, so do $\tilde{\rho}(x, t)=\rho(x, 1-t)$ and $\tilde{v}(x, t)=-v(x, 1-t)$. Furthermore, due to the translational invariance of the matrix chain the final velocity at $t=1$ is opposite to the initial velocity $v_{0}(x)$,

$$
v(x, t=1)=-v(x, t=0) .
$$

This, together with $\rho(x, t=0)=\rho(x, t=1)$, implies that the solutions $\{\tilde{\rho}, \tilde{v}\}$ and $\{\rho, v\}$ coincide at $t=0$. Consequently, these two solutions coincide also at all later times, and (3.9) follows.

The relation between the initial and final velocities, which we needed for the proof, is almost obvious. Indeed, the action $S\left[\rho_{n}, \rho_{n+1}\right]$ is a symmetric functional of the densities $\rho_{n}$ and $\rho_{n+1}$. Therefore if $\rho_{n}=\rho_{n+1}$ the functional variation of $S$ with respect to $\rho_{n}$ equals the variation of $S$ with respect to $\rho_{n+1}$. On the other hand, the end velocity is related to the variation of the action by

$$
\frac{\partial}{\partial x} \frac{\delta S\left[\rho_{n}, \rho_{n+1}\right]}{\delta \rho_{n+1}(x)}=+v(x, t=1)
$$

with a plus sign. On comparison with equation (2.21) we immediately deduce (3.10).

An important consequence of the time reflection symmetry is

$$
v(x, t=1 / 2)=0
$$

In other words, after one half unit of time the fluid completely stops moving. This leads to the following qualitative picture of the droplet evolution. At the initial moment of time $t=0$ the fluid in the vicinity of $x=0$ is moving inwards. The pressure $P=-\pi^{2} \rho^{3} / 3$ acts to slow down and stop this motion. If the initial $\rho(x)$ has been chosen properly then at $t=1 / 2$ the velocity of the fluid vanishes simultaneously everywhere. At larger $t$ the forces of pressure make the droplet move again, repeating backwards in time the evolution from $t=0$ to $t=1 / 2$.

Let us now see how the functional equation (2.29) reproduces the result of (3.8). This equation involves the density of eigenvalues taken at a complex point, $\rho\left[G_{-}(x)\right]$. Such an object is certainly well defined if $\rho(x)$ is analytic. However, the density profile of interest to us, $\rho(x) \propto|x|$ cannot be continued analytically into the complex plane.

The resolution of the arising difficulty is quite simple. One should throw away the absolute value sign and consider instead $\rho(x) \propto x$. Such prescription does yield the correct answer. Indeed, if $\pi \rho(x)=\alpha x$ the functions $G_{+}$and $G_{-}$are given by

$$
G_{ \pm}(x)=\left(1-2 \epsilon^{2} \pm i \alpha\right) x
$$


Then equation (2.29) reduces to

$$
\left(1-2 \epsilon^{2}\right)^{2}+\alpha^{2}=1
$$

which predicts the correct value of $\alpha$

$$
\alpha=2 \epsilon \sqrt{1-\epsilon^{2}} .
$$

This prescription works because no fluid ever flows through $x=0$. In fact, the regions $x>0$ and $x<0$ do not really interact with each other. Furthermore, nothing in Euler equations requires the density to be manifestly positive. For instance, we can flip the sign of $\rho(x, t)$ for $x<0$. The density so obtained, $\rho(x, t) \propto x$ will be analytic in $x$ and will still satisfy Euler equations. On the other hand, the $x>0$ part of the eigenvalue density remains unchanged allowing us to read off the correct answer.

So far the quartic interaction $g \operatorname{tr} M^{4} / 4$ has not played any role in our analysis. An identical effect occurs in the standard formalism of $c=1$ string theory based on matrix quantum mechanics [2] [7]. There, too, one expands the potential around the local maximum and only the quadratic terms matter. In the hydrodynamic picture the interaction terms modify the initial velocity of the droplet by an amount negligible for small $x$. Therefore, for $x \rightarrow 0$ the effects of interaction can be taken into account via perturbation theory. The validity of such perturbative expansion is ensured by the smallness of $x$ even though the coupling $g$ is of order one. In a sense, we are lucky that this perturbation theory is valid precisely in the critical domain.

When the lattice spacing gets bigger than one such a simple picture ceases to be valid. For $\epsilon>1$ imparting the initial velocity $v=-2 \epsilon^{2} x$ to a droplet configuration $\rho(x) \propto|x|$ will always make the droplet collapse. In this case the cubic correction to the initial $v$ given by $g x^{3} / 2$ provides a small, but essential, amount of outward directed velocity that is needed to prevent such collapse.

Quite surprisingly, the interaction effects are not completely trivial even if $\epsilon$ is less than one. This can be seen already in the first order of perturbation theory. Indeed, let us evaluate the first correction to the linear ansatz (3.3). To this end we expand $\rho$ and $v$ in powers of $x$

$$
\left\{\begin{array}{c}
\pi \rho(x, t)=\alpha_{1}(t)|x|+\alpha_{2}(t)|x|^{3}+\ldots \\
v(x, t)=\beta_{1}(t) x+\beta_{2}(t) x^{3}+\ldots
\end{array}\right.
$$

Technically, it is more convenient to work directly with the Hopf function $f=v+i \pi \rho$. For $x>0$ this function has a power series expansion in $x$,

$$
f(x, t)=f_{1}(t) x+f_{2}(t) x^{3}+\ldots .
$$


Substituting this into the Hopf equation (2.26) and collecting the terms of order $x^{3}$ we obtain an ordinary differential equation on $f_{2}(t)$

$$
\dot{f}_{2}+4 f_{2} f_{1}=0
$$

Now we can use the explicit solution for $f_{1}$ given by (3.6) to find

$$
f_{2}(t)=\frac{i c_{2}}{\left(\tau-i \tau_{0}\right)^{4}}
$$

where $\tau \equiv t-1 / 2$ and $c_{2}$ is a constant of integration. At $\tau=0$ we must have $v=0$ which means that $c_{2}$ is real. Further, the boundary condition (3.1) yields $\operatorname{Re} f_{2}(t=0)=g / 2$ fixing $c_{2}$ to be

$$
c_{2}=-\frac{g}{32 \epsilon^{4} \sin (4 \arcsin \epsilon)}=\frac{g}{128 \epsilon^{5}\left(2 \epsilon^{2}-1\right) \sqrt{1-\epsilon^{2}}} .
$$

As a result, we find the first correction to the eigenvalue density

$$
\pi \rho(x)=2 \epsilon \sqrt{1-\epsilon^{2}}|x|-\frac{g|x|^{3}}{2 \tan (4 \arcsin \epsilon)}+\ldots
$$

If $\epsilon=1 / \sqrt{2}$ this correction becomes infinite. The technical reason for that is very clear. When $\epsilon=1 / \sqrt{2}$ the value of $f_{2}(t)$ at $t=0$ is purely imaginary, $f_{2}(0)=-4 i c_{2}$. Therefore, even though we do perturb the eigenvalue density by $x^{3}$ terms, no change in the initial velocity arises. In this way, the $x^{3}$ mode at $\epsilon=1 / \sqrt{2}$ could be viewed as a "resonance" of the droplet. Certainly, a similar picture of resonances should also appear in the equivalent approach to $c=1$ strings based on matrix quantum mechanics.

Whenever such resonances occur the perturbative expansion needs to be modified. It turns out that an adequate modification is given by the following formula

$$
f(x, t)=f_{1}(t) x+f_{2}(t) x^{3} \log x+\tilde{f}_{2}(t) x^{3}+\ldots
$$

For this to be consistent with the Hopf equation $f_{2}(t)$ must satisfy the old differential equation (3.16) while $\tilde{f}_{2}(t)$ should obey

$$
\dot{\tilde{f}}_{2}+4 f_{1} \tilde{f}_{2}+f_{1} f_{2}=0
$$

Consequently, the function $f_{2}(t)$ is still given by formula (3.17) with a real $c_{2}$ and $\tau_{0}=$ $\sqrt{1-\epsilon^{2}} / 2 \epsilon=1 / 2$. However, the constant $c_{2}$ is not fixed by the boundary conditions on $f_{2}(t)$ anymore. Instead, it will be determined from the boundary conditions on $\tilde{f}_{2}$. 
Since the initial velocity $v_{0}(x)$ contains no logarithms we must demand that $\operatorname{Re} f_{2}(t=$ $0)=0$. According to (3.17) this is possible only if $\epsilon=1 / \sqrt{2}$. In other words, it is not always possible to add $x^{3} \log x$ to the eigenvalue density — for that the lattice spacing must take a special value. And, in agreement with the picture of resonances, it is precisely at this special value when the original power series (3.19) fails.

Given an explicit solution for $f_{2}$ (with $c_{2}$ undetermined) we can find $\tilde{f}_{2}$ from (3.21). Imposing the usual conditions $\operatorname{Re} \tilde{f}_{2}(t=1 / 2)=0$ and $\operatorname{Re} \tilde{f}_{2}(t=0)=g / 2$ fixes $c_{2}$ in (3.17) to be $c_{2}=g / 2 \pi$ and leads to the following expression for $\tilde{f}_{2}(t)$

$$
\tilde{f}_{2}(t)=\frac{i}{\left(\frac{1}{2}+i \tau\right)^{4}}\left[-\frac{g}{2 \pi} \log \left(\frac{1}{2}+i \tau\right)+\tilde{c}_{2}\right]
$$

with a yet undetermined constant $\tilde{c}_{2}$. The eigenvalue density which corresponds to such $f$ has a logarithmic singularity at $x=0$

$$
\pi \rho(x)=|x|+\frac{2 g}{\pi}|x|^{3} \log \left(\frac{1}{\lambda|x|}\right)+\mathcal{O}\left(x^{4}\right) .
$$

The scale $\lambda$ is related to $\tilde{c}_{2}$ by $\tilde{c}_{2}=(g / 4 \pi) \log \left(\lambda^{2} / 2\right)$ and cannot be determined from perturbation theory. To find such a scale, as well as the specific numerical value of the critical coupling $g=g_{\mathrm{cr}}(\epsilon)$ one would have to construct the eigenvalue density for all, not only small, $x$ and impose certain conditions on the global analytic structure and normalization of $\rho(x)$. These conditions shall be discussed in more detail at the end of the next section. However, notwithstanding any ambiguity in the value of $\tilde{c}_{2}$ the structure of the singularity in $\rho(x)$ is still fixed uniquely by the Hopf equation.

Similar logarithmic singularities arise also in higher orders. In general, for $\epsilon=\epsilon_{p q} \equiv$ $\sin (\pi p / 2 q)$ the eigenvalue density has a logarithmic correction of order $|x|^{2 q-1} \log [1 /(\lambda|x|)]$. This can be proved in a rather elementary fashion with the aid of the functional equation (2.29). Indeed, if the eigenvalue density does not have logarithms the functions $G_{ \pm}(x)$ can be expanded in a power series

$$
G_{ \pm}(x)=\sum_{k=1}^{\infty} b_{k}^{ \pm} x^{2 k-1}
$$

By construction, $G_{+}$and $G_{-}$are complex conjugate, so that $b_{k}^{+}=\left(b_{k}^{-}\right)^{*}$. Furthermore, since $\operatorname{Re} G_{ \pm}(x)=U^{\prime}(x) / 2$ is a cubic polynomial all $b_{k}^{ \pm}$with $k \geq 3$ are purely imaginary and thus satisfy $b_{k}^{-}=-b_{k}^{+}$.

Let us try to solve the functional equation (2.29) order by order in $x$. In the first order, $b_{1}^{+} b_{1}^{-}=1$ which means $b_{1}^{ \pm}=\exp ( \pm i \varphi)$ with a certain real $\varphi$. Actually, comparing 
(3.24) to (3.13) we see that the angle $\varphi$ is related to the lattice spacing $\epsilon$ by $\cos \varphi=1-2 \epsilon^{2}$ or, equivalently, $\epsilon=\sin (\varphi / 2)$.

If we know all $b_{k}^{ \pm}$with $k \leq q-1$ and wish to find $b_{q}^{ \pm}$we expand $G_{+}\left[G_{-}(x)\right]$ in powers of $x$ and collect the terms of order $x^{2 q-1}$. As a consequence of (2.29) the sum of such terms must vanish. This yields

$$
b_{q}^{+}\left(b_{1}^{-}\right)^{2 q-1}+b_{1}^{+} b_{q}^{-}+\left\{\begin{array}{l}
\text { terms which depend } \\
\text { only on } b_{1}^{ \pm}, \ldots, b_{q-1}^{ \pm}
\end{array}\right\}=0 .
$$

For certain values of $\epsilon$ equation (3.25) degenerates. Indeed, since $b_{q}^{-}=-b_{q}^{+}$and $b_{1}^{ \pm}=$ $\exp ( \pm i \varphi)$ we find that

$$
b_{q}^{+}\left(b_{1}^{-}\right)^{2 q-1}+b_{1}^{+} b_{q}^{-}=-2 i b_{q}^{+} \sin (q \varphi) \mathrm{e}^{-i(q-1) \varphi} .
$$

If $\varphi=\pi p / q$ the variable $b_{q}^{+}$disappears from the right hand side of (3.25). Then, unless the rest of (3.25) vanishes at the same time, we encounter a contradiction. As a consequence, for these values of $\varphi$ or, equivalently, for $\epsilon=\sin (\pi p / 2 q)$ the eigenvalue density develops an additional singularity. We have checked explicitly that this is indeed true in the first seven orders of perturbation theory.

The same type of reasoning demonstrates that the position, the order and the logarithmic nature of arising singularities are preserved if the quartic interaction $V(M)$ is replaced by a more complicated potential. There may be, however, exceptional situations. They occur when the terms enclosed by the curly brackets in equation (3.25) vanish simultaneously with $\sin (q \varphi)$. For that to happen the coefficients of the matrix potential $V(M)$ must be adjusted in a special way. The eigenvalue density which corresponds to such fine-tuned, "multicritical" potentials will exhibit only some, or none, of the above singularities. For instance, in section 5 we shall construct an example of a nonpolynomial potential which gives rise to only one singularity at $\epsilon=1 / \sqrt{2}$. The model with such a potential can be solved exactly. In fact, it is a generalization of the Penner-type model where an exact solution can be gotten independently using the method of loop equations [20].

The above considerations apply when the coupling constant $g$ strictly equals $g_{\mathrm{cr}}(\epsilon)$. Only then $\rho(x) \propto|x|$ around $x=0$. Whenever $g$ is shifted away from $g_{\mathrm{cr}}(\epsilon)$ (or, in the language of continuum theory, when the cosmological constant is given a nonzero value) the eigenvalue density gets perturbed. Such perturbed shape of $\rho(x)$ would be useful to know for several reasons. First, if one wishes to explore the critical behavior of the free energy, say using equation (2.25), one needs to know $\rho(x)$ in a certain interval of couplings 
around $g_{\mathrm{cr}}(\epsilon)$. Further, we shall see that such analysis provides an independent derivation of the logarithmic singularities which were simply guessed in equation (3.20).

For a small nonzero $\delta g=g-g_{\mathrm{cr}}(\epsilon)$ the $|x|$-type singularity in $\rho(x)$ disappears. Instead the eigenvalue density develops a pair of close square root branch points. These branch points merge when $\delta g$ vanishes producing $\rho(x) \propto|x|$. In fact, the analytic structure of exactly the same kind occurs in the $c=1$ matrix model with a continuous target space where the eigenvalue density is given by the WKB formula [2] 21]

$$
\pi \rho(x)=\sqrt{2[E-\tilde{U}(x)]}
$$

with $\tilde{U}(x)=-\tilde{\kappa}^{2} x^{2} / 2+g x^{4} / 4$.

Remarkably, the existence of two square root branch points is fully consistent with Euler equations. To see this let us consider the following "hyperbolic" ansatz for $\rho$ and $v$

$$
\left\{\begin{aligned}
\pi \rho(x, t) & =\sqrt{b(t)+\alpha^{2}(t) x^{2}} \\
v(x, t) & =\beta(t) x
\end{aligned}\right.
$$

This ansatz is consistent with Euler equations provided that $\alpha(t)$ and $\beta(t)$ obey the old equations (3.4) while $b(t)$ satisfies

$$
\dot{b}+2 \beta b=0
$$

Actually, the linear ansatz (3.3) that we considered previously is simply a $b=0$ case of the hyperbolic solution.

The eigenvalue density which correspond to (3.27)

$$
\pi \rho(x)=\sqrt{b(0)+4 \epsilon^{2}\left(1-\epsilon^{2}\right) x^{2}}
$$

has two square root branch points. Moreover, such $\rho(x)$ can be viewed as a generalization of the WKB formula $(3.26)$ to finite nonzero lattice spacings. Indeed, for small $x$ the WKB solution has the form

$$
\pi \rho(x)=\sqrt{2 E+\tilde{\kappa}^{2} x^{2}}
$$

identical to (3.29). The parameter $b \equiv b(0)$ in the hyperbolic solution and the Fermi energy $E$ in (3.26) play extremely similar roles. Both $b$ and $E$ are in fact functions of $\delta g$ which are determined by the normalization condition that the total integral of the full, exact $\rho(x)$ equals one. Obviously, for small coupling deviations $\delta g$ the parameters $b$ and $E$ are also small.

In the critical region of interest to us both $b$ and the eigenvalue magnitude $x$ are small but can be of the same order with respect to each other. That is, if the characteristic 
width of the double well potential $V(M)$ equals $a$ then $x \sim \sqrt{b} \ll a$. It is exactly this region of eigenvalues - a small interval of size $\sim \sqrt{b}$ around the top of the potentialthat is important, for example, in the matrix quantum mechanics representation of $c=1$ strings. For such $x$ and $b$ equation (3.29) yields a good approximation to $\rho(x)$ which can be systematically improved upon.

The corrections to (3.29) can be easily computed using the functional equation $G_{+}\left[G_{-}(x)\right]=x$. We shall look for the corrected eigenvalue density in the form

$$
\pi \rho(x)=\sqrt{b+x^{2} \sin ^{2} \varphi+g r(x)}
$$

where $\varphi$ is defined by $\epsilon=\sin (\varphi / 2)$ and $r(x)$ represents the first correction. For $x^{2} \sim b$ the quantity $r(x)$ should be small of order $b^{2}$ or, equivalently, $x^{4}$. Actually, for the special case of $b=0$ the estimate $r(x) \sim x^{4}$ follows already from (3.19). The functions $G_{+}$and $G_{-}$are then

$$
G_{ \pm}(x)=x \cos \varphi+\frac{g x^{3}}{2}+i \sqrt{b+x^{2} \sin ^{2} \varphi+g r(x)} .
$$

The approximation we are constructing is formally the same as the power series expansion in $g$. Indeed, in such an expansion each extra power of $g$ comes along with either $b$ or $x^{2}$. For example, $g r(x) \sim g b^{2}$ is much smaller than $b+x^{2} \sin ^{2} \varphi$ which is of order $b$. The same applies to $g x^{3} / 2 \sim g b x$ which is much smaller than $x \cos \varphi$. In short, the true parameter of our expansion is $g b \sim g x^{2} \ll 1$ so that we can formally expand in $g$ even though $g$ by itself is not small.

Doing this in (3.31) to first order in $g$ yields

$$
G_{ \pm}(x)=G_{ \pm}^{(0)}(x)+g H_{ \pm}(x)
$$

with

$$
G_{ \pm}^{(0)}(x)=x \cos \varphi \pm i \sqrt{b+x^{2} \sin ^{2} \varphi}
$$

and

$$
H_{ \pm}(x)=\frac{x^{3}}{2} \pm i \frac{r(x)}{2 \sqrt{b+x^{2} \sin ^{2} \varphi}} .
$$

Then the functional equation (2.29) expanded to $\mathcal{O}\left(g^{2}\right)$ reduces to

$$
G_{+}^{(0)}\left[G_{-}^{(0)}(x)\right] H_{-}(x)+H_{+}\left[G_{-}^{(0)}(x)\right]=0 .
$$

We now substitute for $G_{ \pm}^{(0)}$ and $H_{ \pm}$the explicit expressions of (3.32) and (3.33). This produces the following linear functional equation on $r(x)$

$$
r(y)-r(x)=x y^{3}-x^{3} y+\left(x^{4}-y^{4}\right) \cos \varphi
$$


where the letter $y$ stands for the combination

$$
y \equiv x \cos \varphi-i \sqrt{b+x^{2} \sin ^{2} \varphi}
$$

When $x^{2} \sim y^{2} \sim b$ both sides of this equation are of order $b^{2}$. It is therefore natural to look for a solution of (3.35) in terms of a polynomial "homogeneous" in $x^{2}$ and $b$

$$
r(x)=R_{1} x^{2} b+R_{2} x^{4} .
$$

Upon a certain amount of computation one finds that this form of $r$ is indeed consistent with the functional equation (3.35) provided $R_{1}$ and $R_{2}$ equal

$$
\begin{aligned}
& R_{1}=\frac{1}{2 \cos \varphi}=\frac{1}{2\left(1-2 \epsilon^{2}\right)} \\
& R_{2}=\frac{1}{2 \cos \varphi}-\cos \varphi=\frac{1}{2\left(1-2 \epsilon^{2}\right)}-1+2 \epsilon^{2} .
\end{aligned}
$$

Expressions (3.36) and (3.37) generalize the perturbative expansion (3.19) to the case of a nonzero cosmological constant. Not surprisingly, these expressions also develop poles at $\epsilon=1 / \sqrt{2}$, where the polynomial form for $r(x)$ ceases to be a solution of (3.35). However, even for $\epsilon=1 / \sqrt{2}$ the equation on $r(x)$ can still be solved. Remarkably, the solution will produce a logarithmic contribution in $r$ automatically without adding it by hand as we did before in (3.20).

When $\epsilon=1 / \sqrt{2}$ our linear functional equation becomes

$$
\left\{\begin{array}{l}
r(y)-r(x)=y^{3} x-x^{3} y \\
y \equiv-i \sqrt{b+x^{2}}
\end{array}\right.
$$

We are interested in the solution of this equation which has two square root branch points at $x= \pm i \sqrt{b}$ and, on the real axis, is an even function of $x$ regular at $x=0$.

Such a solution is defined on a complex plane with two cuts as shown in the figure and can be found using the method of dispersion relations 1 . Indeed, the imaginary part of $r(x)$ on the edges of the cuts follows directly from (3.38) and equals

$$
\operatorname{Im} r(-i u+0)=u\left(2 u^{2}-b\right) \sqrt{u^{2}-b} .
$$

To see this, consider equation (3.38) with a positive real $x$. Then $y=-i u=-i \sqrt{b+x^{2}}$ is positioned on the right edge of the lower cut. Furthermore, $\operatorname{Im} r(x)=0$. As a consequence, $\operatorname{Im} r(y)=\operatorname{Im}\left(y^{3} x-x^{3} y\right)$ leading immediately to (3.39).

Once the imaginary part of $r$ on any of the four cut edges has been found the imaginary part on the other three edges can be determined by analytic continuation. Then we can write down a dispersion relation for the function $r(x)$ to fix its values on the real axis.

4 A shorter way to solve this equation, suggested by J. Goldstone, is to use the substitution $x=$ $\sqrt{b} \sinh \psi$. Nonetheless, the solution based on dispersion relations will be necessary to illustrate several important points later, in section 4. 


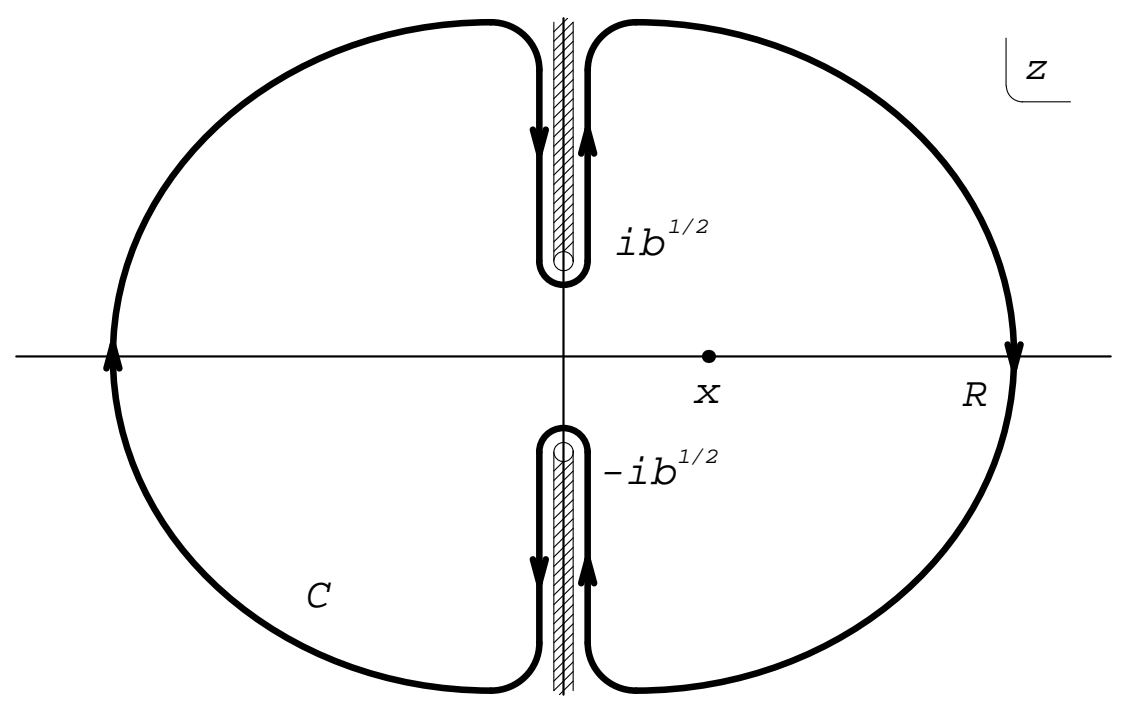

Fig. 3: The cut structure of the eigenvalue density in the critical region and the contour $C$ used in the dispersion relation. The same cuts and integration contour will arise in our analysis of the Kosterlitz-Thouless phase transition in section 4.

To this effect consider the integral

$$
I(x)=\oint \frac{r(z) d z}{z^{5}(x-z)}
$$

taken along the contour $C$ shown in the figure. The large radius loops closing $C$ at infinity do not make any contribution to this integral. This follows from the nature of our approximation. Indeed, let us choose the loop radius $R$ to be much larger than $b$ but still much smaller than the characteristic size of the potential $V(M)$. For $|z|<R$ the function $r(z)$ depends only on $z$ and $b$ and has the general scaling form $r(z)=b^{2} s\left(z^{2} / b\right)$. However, for $z^{2} \gg b$ the leading asymptotic behavior of $r(z)$ should be independent of $b$, simply because $r(z)$ has a finite limit when $b \rightarrow 0$, given by (3.23). Thus for large $\xi=z^{2} / b$ the function $s$ behaves as $s(\xi) \sim \xi^{2}$ so that $r(z) \sim z^{4}$, perhaps with logarithmic corrections. As a consequence for large $z$ the integrand in (3.40) decays as $1 / z^{2}$ and the contribution from infinity equals zero.

Therefore, the only parts of the contour that contribute to $I(x)$ are the four cut edges. In fact, due to the square root nature of the branch points the real part of $r$ on the cuts 
cancels out of the integral so that

$$
\begin{aligned}
I(x) & =\int_{b}^{+\infty}\left\{\frac{i \delta r(i u)}{(i u)^{5}(x-i u)}+\frac{i \delta r(-i u)}{(-i u)^{5}(x+i u)}\right\} d u \\
& =-4 i x \int_{b}^{+\infty} \frac{d u}{u^{4}} \frac{\left(2 u^{2}-b\right) \sqrt{u^{2}-b}}{x^{2}+u^{2}}
\end{aligned}
$$

where

$$
\delta r(i u)=-\delta r(-i u)=-2 i u\left(2 u^{2}-b\right) \sqrt{u^{2}-b}
$$

is the jump of the function $r(z)$ across the cut.

Alternatively, $I(x)$ can be evaluated by taking residues at $z=0$ and $z=x$. This yields

$$
I(x)=2 \pi i \frac{r(x)}{x^{5}}-2 \pi i\left(\frac{\tilde{r}_{0}}{x^{5}}+\frac{\tilde{r}_{1}}{x^{3}}+\frac{\tilde{r}_{2}}{x}\right)
$$

the numbers $\tilde{r}_{0}, \tilde{r}_{1}$ and $\tilde{r}_{2}$ being defined by $r(x)=\tilde{r}_{0}+\tilde{r}_{1} x^{2}+\tilde{r}_{2} x^{4}+\mathcal{O}\left(x^{6}\right)$. This allows us to compute $r(x)$. Evaluating the integral in (3.41) and comparing the result to (3.42) we easily deduce

$$
r(x)=r_{0}+r_{1} x^{2}+r_{2} x^{4}-\frac{x}{\pi}\left(2 x^{2}+b\right) \sqrt{x^{2}+b} \log \left[\frac{\sqrt{x^{2}+b}+x}{\sqrt{x^{2}+b}-x}\right]
$$

The new constants $r_{0}, r_{1}$ and $r_{2}$ which appear in (3.43) are related to the residues $\tilde{r}_{0}, \tilde{r}_{1}$ and $\tilde{r}_{2}$ according to the formulas

$$
\left\{\begin{array}{l}
r_{0}=\tilde{r}_{0} \\
r_{1}=\tilde{r}_{1}+2 b / \pi \\
r_{2}=\tilde{r}_{2}+14 / 3 \pi .
\end{array}\right.
$$

At this point it is necessary to check that the expression we obtained satisfies the full functional equation (3.38). The reason is that in the above analysis we threw out the real part of the functional equation taking only its imaginary part. It turns out that the full functional equation is obeyed if we impose an additional constraint $r_{1}=r_{2} b$. Thus we finally get

$$
r(x)=r_{0}+r_{2} x^{2}\left(x^{2}+b\right)-\frac{x}{\pi}\left(2 x^{2}+b\right) \sqrt{x^{2}+b} \log \left[\frac{\sqrt{x^{2}+b}+x}{\sqrt{x^{2}+b}-x}\right] .
$$


The parameter $r_{0}=r(0)$ can be set to zero by adequately redefining $b$. The parameter $r_{2}$ plays the role analogous to $\tilde{c}_{2}$ in (3.23) and for the same reason cannot be determined by expansion methods. Note though that this parameter controls a totally regular, analytic contribution. The nonanalytic part of $r(x)$ is, on the contrary, fully unambiguous.

If we take $b$ to zero we recover the logarithmic singularity in $\rho(x)$ already found in (3.23). Indeed, given $r_{0}=0$ the small $b$ limit of (3.44) becomes

$$
r(x)=r_{0}+r_{2} x^{4}-\frac{2 x^{4}}{\pi} \log \left(\frac{4 x^{2}}{b}\right)
$$

which is fully consistent with (3.23) provided $r_{2}=-(2 / \pi) \log \left(\lambda^{2} b / 4\right)$.

The expressions for the eigenvalue density we found in this section can be used, in principle, to determine the critical behavior of the free energy. We shall pursue this problem elsewhere. Furthermore, our expressions indicate that the analytic structure of the full exact solution for the matrix chain is rather complex. When viewed as an analytic function of the complex variable $\epsilon$ this solution would have singularities at all points of the form $\epsilon_{p q}=\sin (\pi p / 2 q)$ (and, as a consequence, at all other $\epsilon \in[0,1]$.) Remarkably, certain elliptic functions, like the Dedekind function

$$
\mathcal{D}^{-1}(q)=q^{-1 / 24} \prod_{n=1}^{\infty}\left(1-q^{n}\right)^{-1}
$$

with $q=\exp (4 i \arcsin \epsilon)$ have an analogous singularity structure. It would be quite interesting indeed if the exact $\rho(x)$ for the matrix chain was actually connected to such elliptic functions.

\section{Kosterlitz-Thouless Phase Transition in Infinite Matrix Chain}

Mathematically, the Kosterlitz-Thouless phase transition in the matrix chain is quite similar to the logarithmic singularities we have discussed in the previous section. This transition occurs at the point $\epsilon=1$ where the small $x$ expansion for the eigenvalue density given by (3.8) or (3.19) ceases to make sense.

In this section we shall demonstrate that the critical behavior of the eigenvalue density at $\epsilon=1$ is given by a logarithmic law

$$
\rho(x) \propto \frac{|x|}{\log [1 /(\lambda|x|)] .}
$$

Such behavior is somewhat atypical. Indeed, in matrix models the leading singularity in $\rho(x)$ is usually powerlike, $\rho(x) \propto|x|^{\delta}$. However, it is possible to prove that for $\epsilon=1$ 
a power law would be inconsistent with the saddle point equation. Although the explicit proof shall not be given here one can easily reconstruct it using the methods of this section.

We can acquire some idea of what $\rho(x)$ should be by inspecting the $\epsilon<1$ solution (3.8). As $\epsilon \rightarrow 1$ the slope $\alpha$ in $\rho(x) \propto \alpha|x|$ tends to zero. This suggests that at $\epsilon=1$ and $x \rightarrow 0$ the density $\rho(x)$ should vanish faster than $\alpha|x|$ with any $\alpha$. On the other hand, when $\epsilon=1$ the asymptotics $\rho(x) \propto|x|^{\delta}$ with $\delta>1$ contradicts Euler equations. Therefore, it is natural to look for $\rho(x)$ which vanishes faster than $|x|$ but slower than $|x|^{\delta}$ for any $\delta>1$.

Expression (4.1) has exactly this property and, most importantly, is consistent with Euler equations. The most convenient way to see this is to use the functional equation (2.29). At $\epsilon=1$ the functions $G_{+}(x)$ and $G_{-}(x)$ are

$$
G_{ \pm}(x)=-x+\frac{g x^{3}}{2} \pm i \pi \rho(x) .
$$

Such form for $G_{ \pm}$is very special. Indeed, if we neglect both the $g x^{3} / 2$ and $i \pi \rho(x)$ terms altogether the resulting functions $G_{ \pm}(x)=-x$ would nonetheless satisfy $G_{+}\left[G_{-}(x)\right]=x$. From this point of view, the Kosterlitz-Thouless phase transition is just the most senior of the logarithmic singularities found in section 3. At these singularities, which occur for $\epsilon=\sin (\pi p / 2 q)$, the small $x$ behavior of $G_{+}$and $G_{-}$is given by (3.13), or

$$
G_{ \pm}(x)=\mathrm{e}^{ \pm i \pi p / q} x+\mathcal{O}\left[x^{3} \log \left(\frac{1}{\lambda x}\right)\right] .
$$

If we raise $G_{+}(x)$ or $G_{-}(x)$ to the functional power $2 q$-that is, compose $G_{+}(x)$ with itself $2 q$ times, we shall get

$$
\underbrace{G_{+}\left[G_{+}\left[\ldots G_{+}(x)\right] \ldots\right]}_{2 q \text { times }}=x+\ldots
$$

One could say that, up to the higher order terms, the functions $G_{ \pm}$are the fixed points of order $2 q$ with respect to the operation of functional composition. All the logarithmic singularities of the matrix chain are in one-to-one correspondence with such fixed points. In this language, the Kosterlitz-Thouless phase transition is represented by the fixed point of the lowest - second-order. Consequently, there should be no surprise that at $\epsilon=1$ we also encounter the logarithmic type of scaling behavior.

Let us now add $\pi \rho(x)=A|x| / \log [1 /(\lambda|x|)]$ with an unknown coefficient $A$ and expand $G_{+}\left[G_{-}(x)\right]$ around $x=0$. In doing this expansion we shall use again the prescription which directs us to throw away all absolute value signs. The justification for that will be given later. In short, we write

$$
G_{ \pm}(x)=-x \mp \frac{i A x}{\log (\lambda x)}+\ldots
$$


The terms denoted by dots include the $g x^{3} / 2$ term coming from the interaction potential and the higher order corrections to the eigenvalue density. Generally, we expect these corrections to be down by powers of $1 /|\log (\lambda x)|$ or $\log |\log (\lambda x)| /|\log (\lambda x)|$, like the higher order terms in the series (2.35). Expanding the right hand side of the functional equation around $x=0$ yields

$$
\begin{aligned}
G_{+}\left[G_{-}(x)\right] & =-G_{-}(x)-\frac{i A G_{-}(x)}{\log \left[\lambda G_{-}(x)\right]}+\ldots \\
& =x-i A x \log (\lambda x)+i A x \log (-\lambda x)\left[1-\frac{i A}{\log (\lambda x)}\right]+\ldots
\end{aligned}
$$

We can now replace $\log (-\lambda x)=\log (\lambda x)-i \pi$ and expand (4.4) in powers of $1 /|\log (\lambda x)|$ to get

$$
G_{+}\left[G_{-}(x)\right]=x+\frac{A(A-\pi) x}{\log ^{2}(\lambda x)}+\mathcal{O}\left(\frac{x}{\log ^{3} \lambda x}\right) .
$$

Therefore, the functional equation $G_{+}\left[G_{-}(x)\right]=x$ is indeed satisfied to the leading order provided $A=\pi$, in complete agreement with (4.1).

Note that in the last line of (4.4) we kept the terms of order $x / \log ^{2}(\lambda x)$. At first sight this may seem redundant and even incorrect. After all, exactly such terms have been completely neglected in the original expression for $\rho(x)$ given by (4.1). Nonetheless, these additional terms change only the next, $\mathcal{O}\left[x /\left|\log ^{3}(\lambda x)\right|\right]$ order of expansion. They cancel out from the $x / \log ^{2}(\lambda x)$ part of the series very much like the leading term itself, $\rho(x) \propto x /|\log (\lambda x)|$ contributes only $x / \log ^{2}(\lambda x)$ and not $x /|\log (\lambda x)|$ to the right hand side of $(4.5)$.

At this point we would like to show how one constructs the solution of Euler equations, in terms of $v$ and $\rho$ or the Hopf function $f$, which corresponds to (4.1). There are three reasons why this is required. First, it will provide us with a method to compute all logarithmic corrections to (4.1), prove their universality and sum the correction series in (2.35) all at once. Second, we shall be able to justify our way of dealing with absolute value signs in (4.4). Third, functional equations like (2.29) can have spurious solutions which must be detected and eliminated. An example of such spurious solution is given by a power series

$$
G_{ \pm}(x)=-x+\frac{g x^{3}}{2} \pm i \sum_{k=1}^{\infty} a_{k} x^{2 k}
$$

with real coefficients $a_{k}$. By expanding $G_{+}\left[G_{-}(x)\right]$ in powers of $x$ and equating the result to $x$ one can find all $a_{k}$ in a recursive fashion without encountering apparent contradictions. Nonetheless, there is no solution of Euler equations which would give

$$
\pi \rho(x, t=0)=\pi \rho(x, t=1)=\sum_{k=1}^{\infty} a_{k} x^{2 k}
$$


while obeying the boundary condition $v(x, t=0)=-2 x+g x^{3} / 2$. In other words, a solution of the functional equation (2.29) does not always yield a solution of the full hydrodynamic boundary problem (2.16), (2.24). The reason is, when viewed as an analytic function of the complex variable $x$ the eigenvalue density $\rho(x)$ may have several different regular branches. One of them takes real values for real $x$ and represents the actual eigenvalue density of the infinite matrix chain. The power series expansions we construct refer, by definition, only to this branch. However, the functional equation (2.29) involves the eigenvalue density computed at a complex point, $\rho\left[G_{-}(x)\right]$ which might take us to an entirely different branch of $\rho$. The relationship between this other branch and the "proper" branch expandable in a series can in reality be very complicated.

We can check whether a given solution of (2.29) suffers from branch choice problems by computing $v$ and $\rho$ at $t=1 / 2$. A solution which is consistent with the complete set of hydrodynamic equations (2.16), (2.24) shall have $v(x, t=1 / 2)=0$ as required by the time reflection symmetry (3.12). Let us prove that this is indeed the case for the logarithmic eigenvalue density (4.1). To this end we shall use the implicit solution of the Hopf equation given by formula (2.27). For $\epsilon=1$ the initial velocity equals $v_{0}(x)=-2 x+\mathcal{O}\left(x^{3}\right)$ so that the initial value of the Hopf function is

$$
f_{0}(x)=-2 x+\frac{i \pi x}{\log [1 /(\lambda x)]}+\ldots
$$

Then the value of $f$ at $t=1 / 2$, denoted $f_{1 / 2}(x)$ can be determined from the equation

$$
\frac{2 i x}{\pi} \log \left\{\lambda\left[x-\frac{1}{2} f_{1 / 2}(x)\right]\right\}=x-\frac{1}{2} f_{1 / 2}(x)
$$

We are interested in solving this transcendental equation only in the limit of small $x$. It is easy to check that the relevant solution is given by

$$
f_{1 / 2}(x)=\frac{4 i}{\pi} x \log \left(\frac{1}{\tilde{\lambda} x}\right)+\ldots
$$

where the dots stand for the subleading corrections of order $\mathcal{O}[x \log |\log (\tilde{\lambda} x)|]$. Indeed, for such $f_{1 / 2}$

$$
\begin{aligned}
\log \left\{\lambda\left[x-\frac{1}{2} f_{1 / 2}(x)\right]\right\} & =\log (\lambda x)-\frac{\pi i}{2}+\log \left[\frac{2}{\pi} \log \left(\frac{1}{\tilde{\lambda} x}\right)-1\right] \\
& =\log x-\frac{\pi i}{2}+\mathcal{O}[x \log |\log x|]
\end{aligned}
$$


where we neglected the real valued terms which involve double logarithms as well as real constant terms. The imaginary constants, on the other hand, can be kept for the reason explained after equation (4.5). In this approximation the left hand side of (4.8) equals

$$
\frac{2 i x}{\pi}\left(\log x-\frac{\pi i}{2}\right)=x-\frac{2 i x}{\pi} \log \left(\frac{1}{x}\right)
$$

which up to the terms of order $\mathcal{O}[x \log |\log (\tilde{\lambda} x)|]$ coincides with $x-f_{1 / 2}(x) / 2$.

The expression for $f_{1 / 2}$ in (4.9) is purely imaginary. Therefore, the fluid velocity at $t=1 / 2$, given by the real part of $f_{1 / 2}$, does vanish. Let us now examine the corresponding fluid density,

$$
\pi \rho_{1 / 2}(x)=\frac{4 x}{\pi} \log \left(\frac{1}{\tilde{\lambda} x}\right)+\ldots
$$

Naively, we could expect that this expression receives logarithmic corrections, generally of order $\mathcal{O}[x \log |\log (\tilde{\lambda} x)|]$. However, in a most remarkable way all such corrections happen to cancel. To avoid any misunderstanding, it would be false to say that the matrix chain eigenvalue density itself does not get logarithmic corrections. It does. But the quantity $\rho_{1 / 2}(x)$ is still very simple, much simpler than $\rho(x)$. In particular, the infinity of logarithmic terms in the double series (2.35) are fully summarized by equation (4.10). That is to say, the only corrections to $(4.10)$ are the $\mathcal{O}\left(x^{3}\right)$ terms due to the quartic interaction in the matrix potential.

To prove this we shall turn our arguments around. Imagine starting at $t=1 / 2$ with a droplet of the density given by $(4.10)$ and zero velocity. Let the droplet move according to Euler equations. Then compute the density and velocity of the droplet at $t=1$.

Certainly, by time reflection we can always reverse the droplet motion to reconstruct the $0 \leq t \leq 1 / 2$ part of the trajectory. As a result the boundary condition $\rho(x, t=0)=$ $\rho(x, t=1)$ will be satisfied automatically. The only remaining boundary condition one will have to check is the equation on velocity

$$
v(x, t=0)=-v(x, t=1)=-2 x+\frac{g x^{3}}{2} .
$$

We shall now prove that, neglecting $\mathcal{O}\left(x^{3}\right)$ terms, the logarithmic density of (4.10) generates precisely such a velocity. The density $\rho(x) \equiv \rho(x, t=1)$ that we shall find in the course of this proof will then be the matrix chain eigenvalue density at the Kosterlitz-Thouless point.

The velocity condition (4.11) can be checked conveniently using the implicit Hopf solution (2.27). We start at $t=1 / 2$ with the Hopf function

$$
f_{1 / 2}(x)=\frac{4 i x}{\pi} \log \left(\frac{1}{\tilde{\lambda} x}\right)+\mathcal{O}\left(x^{3}\right)
$$


and we must finish at $t=1$ with $f$ being equal

$$
f_{1}(x) \equiv f(x, t=1)=2 x+i \pi \rho(x)+\mathcal{O}\left(x^{3}\right) .
$$

Since the elapsing time interval is $\Delta t=1 / 2$ the implicit solution (2.27) imposes the following relationship between $f_{1 / 2}$ and $f_{1}$

$$
f_{1}(x)=f_{1 / 2}\left[x-\frac{1}{2} f_{1}(x)\right]
$$

This is a complex valued equation which has both real and imaginary parts. However, the only unknown in $f_{1 / 2}$ and $f_{1}$ is one real valued function $\rho(x)$. Therefore, the internal consistency of equation (4.14) is not automatically guaranteed. Rather, such consistency is an indication of the correct choice for $\rho_{1 / 2}(x)$ that we made in $(4.10)$.

For the purpose of computational convenience let us represent $\rho(x)$ in the form

$$
\pi \rho(x)=\pi|x| \sigma(x)+\mathcal{O}\left(x^{3}\right) .
$$

Note that again the $x<0$ and $x>0$ regions do not interact. Therefore, we can safely replace $|x| \rightarrow x$ in (4.15) so long as we apply our answers only to $x>0$. Then taking (4.12) and (4.13) into account, equation (4.14) translates into

$$
2 x+i \pi x \sigma(x)=-2 x \sigma(x) \log \left[-\frac{i \pi}{2} \tilde{\lambda} x \sigma(x)\right] \text {. }
$$

Using $\log (-i y)=\log y-i \pi / 2$ we see that the imaginary part of this equation is satisfied identically. The real part yields then an equation on $\sigma(x)$,

$$
2 x=-2 x \sigma(x) \log \left[\frac{\pi}{2} \tilde{\lambda} x \sigma(x)\right]
$$

or, equivalently,

$$
\frac{1}{\sigma(x)}+\log \sigma(x)=\log \left(\frac{1}{\lambda x}\right)
$$

with $\lambda=\pi \tilde{\lambda} / 2$. For small $x$ the solution of (4.16) is approximately

$$
\sigma(x) \propto \frac{1}{\log [1 /(\lambda x)]}
$$

in complete agreement with (4.1).

A remarkable property of equation (4.16) is its universality. Indeed, the interaction potential enters $\rho(x)$ only through the $\mathcal{O}\left(x^{3}\right)$ terms which did not matter in our analysis. 
Therefore, the logarithmic terms summarized by $\sigma(x)$ will remain the same for all, not only quartic, matrix interactions. Such universality may indicate that equation (4.16) contains certain information about the continuum limit of the theory. To understand what precisely this information is would be very interesting. A more specific problem that can hopefully be addressed using (4.16) is to find the free energy at $\epsilon=1$. Once we know the critical index of the free energy $\gamma_{\text {str }}$ we can find the effective central charge at $\epsilon=1$ and determine what kind of a conformal field theory the matrix chain describes at that point. If the relation between $\gamma_{\text {str }}$ and the eigenvalue index $\delta$,

$$
\gamma_{\text {str }}=1-\delta
$$

holds in this case, formula (4.1) indicates that at $\epsilon=1$ the string susceptibility is most likely zero. This is characteristic of $c=1$ theories. However, the logarithmic corrections to the free energy at $\epsilon=1$ would certainly be different from the logarithmic corrections in the $c=1$ theory on a straight continuous line. Therefore, these corrections deserve more investigation. For example, equation (4.16) may have an interpretation in terms of vortices which start forming a strongly interacting system precisely at the point $\epsilon=1$.

To compute the free energy carefully one must know the eigenvalue density in a certain interval of coupling deviations $\delta g=g-g_{\mathrm{cr}}$. For small values of $\delta g$ the eigenvalue density can be found, as before, using the method of dispersion relations.

Again, we shall look for $\rho(x)$ which has two close square root branch points at $x \sim$ $\pm i \sqrt{b}$ and is an even real function for real values of $x$. We shall seek an approximation to $\rho$ valid for $x \sim \sqrt{b} \ll a$ where $a$ is the typical width of the quartic potential. Moreover, since the density $\rho_{1 / 2}(x)$ seems to have a simpler form than $\rho(x)$ itself we shall continue working directly with $\rho_{1 / 2}(x)$ even for $b \neq 0$.

The eigenvalue density with the desired properties can be found as a series in powers of $\eta=\sqrt{b} / x$. That is, we go to the region $\sqrt{b} \ll x \ll a$ which is still within the limits of our approximation. In this region $\eta \ll 1$ is a good expansion parameter. Consequently, we may look for $\rho_{1 / 2}(x)$ in terms of a series in powers of $\eta$ or, equivalently, of $b$. We shall now demonstrate that the relevant, consistent with the hydrodynamic picture series is given by

$$
\rho_{1 / 2}(x)=\left.\rho_{1 / 2}(x)\right|_{b=0}+\sum_{k=1}^{\infty} a_{k} \frac{b^{k}}{|x|^{2 k-1}} .
$$

This ansatz for $\rho_{1 / 2}(x)$ is not arbitrary. A similar expansion arises, for example, in the $\epsilon<1$ phase of the matrix chain. There $\rho(x) \sim \sqrt{b+\alpha^{2} x^{2}}$ can also be expanded in a series

$$
\pi \rho(x)=\alpha|x| \sqrt{1+\frac{b}{\alpha^{2} x^{2}}}=\alpha|x|+\sum_{k=1}^{\infty}\left(\begin{array}{c}
1 / 2 \\
k
\end{array}\right) \frac{b^{k}}{(\alpha|x|)^{2 k-1}}
$$


identical in form to (4.17). Both of these expansions are explicitly singular at $x=0$ but the singularity disappears when the full series is summed.

Most interestingly, expansion (4.17) is consistent with the Hopf equation and the boundary conditions for any set of real coefficients $a_{k}$. To see this we simply repeat the computation of equations (4.12) - 4.16). The function $f_{1}(x)=f(x, t=1)$ is still given by equation (4.13) while for $f_{1 / 2}(x)$ we now have

$$
f_{1 / 2}(x)=\frac{4 i}{\pi} x \log \left(\frac{1}{\tilde{\lambda} x}\right)+i \pi \sum_{k=1}^{\infty} a_{k} \frac{b^{k}}{x^{2 k-1}}+\mathcal{O}\left(x^{3}\right) .
$$

Imposing the implicit relation (4.14) we find that again the imaginary part of (4.14) is satisfied identically while the real part yields an equation on $\rho(x)$,

$$
2 x=-2 \rho(x) \log \left[\frac{\pi}{2} \tilde{\lambda} \rho(x)\right]+\frac{\pi^{2}}{2} \rho(x) \sum_{k=1}^{\infty}(-)^{k} a_{k}\left[\frac{4 b}{\pi^{2} \rho^{2}(x)}\right]^{k} .
$$

The coefficients $a_{k}$ have been left undetermined by the Hopf equation. Rather, they are constrained by the analytic structure of $\rho_{1 / 2}(x)$. Indeed, if $\rho_{1 / 2}(x)$ has two cuts shown in fig.3 then the real part of $\rho_{1 / 2}$ on the cut edges follows directly from (4.17). For example, on the right edge of the upper cut, where $x=i u+0$

$$
\begin{aligned}
r(i u) \equiv \operatorname{Re}\left[\pi \rho_{1 / 2}(x)\right] & =\operatorname{Re}\left[\frac{4 x}{\pi} \log \left(\frac{1}{\tilde{\lambda} x}\right)\right] \\
& =2 u .
\end{aligned}
$$

Using the square root nature of the branch points we can then perform an analytic continuation and find the real part of $\rho_{1 / 2}$ on the other three edges.

The whole $\pi \rho_{1 / 2}(x)$ can now be restored through a subtracted dispersion relation as in (3.40). The contour of integration is the same contour $C$ in fig.3 while the dispersion integral itself is a little different,

$$
J(x)=\oint_{C} \frac{\pi \rho_{1 / 2}(z)}{z \sqrt{z^{2}+b}} \frac{d z}{x-z} .
$$

The integrand in (4.21) has been constructed in such a way that the contribution from infinite loops closing the contour $C$ vanishes (see (4.17)) while the cuts contribute only through the real part of $\pi \rho_{1 / 2}(i u)$,

$$
\begin{aligned}
J(x) & =\int_{b}^{+\infty} \frac{2 i r(i u)}{(i u)\left(i \sqrt{u^{2}-b}\right)}\left(\frac{1}{x-i u}+\frac{1}{x+i u}\right) d u \\
& =-8 i x \int_{b}^{+\infty} \frac{d u}{\left(x^{2}+u^{2}\right) \sqrt{u^{2}-b}} .
\end{aligned}
$$


On the other hand, the integral in (4.21) can be evaluated via residues to give

$$
J(x)=-\frac{2 \pi i}{\sqrt{b} x} r_{0}+\frac{2 \pi i}{x \sqrt{x^{2}+b}} \pi \rho_{1 / 2}(x)
$$

where $r_{0}=\pi \rho_{1 / 2}(x=0)$. Comparing this to (4.22) and evaluating the integrals we find

$$
\pi \rho_{1 / 2}(x)=\frac{r_{0}}{\sqrt{b}} \sqrt{x^{2}+b}-\frac{2 x}{\pi} \log \left[\frac{\sqrt{x^{2}+b}+x}{\sqrt{x^{2}+b}-x}\right]
$$

The constant $r_{0}$, similarly to $r_{2}$ in (3.44) cannot be determined by considering the region $x \sim \sqrt{b} \ll a$. To fix $r_{0}$ one would need to construct $\rho_{1 / 2}$ for all, not only small values of $x$. The analytic structure of such globally constructed $\rho(x)$ would include two other branch points at $x= \pm x_{0} \sim a$. Indeed, in matrix models with polynomial potentials the eigenvalue density is usually nonzero only within a finite interval $\left[-x_{0}, x_{0}\right]$. The endpoints $x_{0}$ are, as a rule, square root branch points. From the viewpoint of small $x$ expansions, the square root character of the outer branch points is a nontrivial property of the eigenvalue distribution which is not automatically guaranteed. Therefore, imposing it is likely to constrain the additional parameters such as $r_{0}$ in (4.24) or $r_{2}$ in (3.44). After all, a very similar phenomenon has occured when we imposed the two cut structure on $\rho_{1 / 2}(x)$. There requiring two square root branch points at $x= \pm i \sqrt{b}$ fixed at once an infinite number of coefficients $a_{k}$ which remained unconstrained by the Hopf equation.

To summarize, when the Hopf equation or the functional equation $G_{+}\left[G_{-}(x)\right]=x$

are combined with reasonable assumptions about the analytic structure of the eigenvalue density the solution becomes determined uniquely. In the next section we shall explain, using an example, how such global solutions can be constructed.

\section{Exactly Solvable Interaction Potentials}

By now we have seen that the Euler equations, the Hopf equation and the functional equation (2.29) are all very useful in studying the singularities of $\rho(x)$. In this section we shall show how the same equations can be utilized to construct certain "exactly solvable" potentials $V(M)$ for which $\rho(x)$ is an elementary function. We shall demonstrate that for these potentials the expansion methods of sections 3 and 4 do reproduce the exact results.

The basic idea of such exact solutions is very simple. One chooses at will a midway eigenvalue density $\rho_{1 / 2}(x)$ making sure it is properly normalized. Then the fluid is allowed to evolve under Euler equations. Finally, at $t=1$ one reads off the velocity $v(x, t=1)$ and 
the corresponding eigenvalue density $\rho(x, t=1) \equiv \rho(x)$. The result is an exact solutiongiven by $\rho(x)$ — for the model where the potential can be found from $(3.1),(3.10)$

$$
U^{\prime}(x)=2[x-v(x, t=1)]
$$

The hardest part of the problem is to choose $\rho_{1 / 2}(x)$ correctly, so that the resulting $U(x)$ is physically reasonable. Here we shall present a construction [16] which produces a Pennertype double-well potential rather similar to the pure quartic potential of sections 3 and 4 .

To this effect consider a special $\rho_{1 / 2}(x)$ defined by the following quadratic equation

$$
k\left(x^{2}+r^{2}\right)^{2}+m^{2}\left(x^{2}+r^{2}\right)-2\left(x^{2}-r^{2}\right)+b=0
$$

where $r \equiv \pi \rho_{1 / 2}(x) / 2$. This equation looks, of course, very artificial, and it is. As it turns out, the potential $U(x)$ corresponding to such a choice of $\rho_{1 / 2}$ is particularly simple. Later on, we shall replace the left hand side of (5.2) by a more general polynomial to get a solution for the $\epsilon>1$ phase of the matrix chain.

The potential $U$ and the eigenvalue density $\rho$ that follow from (5.2) can be easily found. As in (4.12) - (4.14) we use the implicit Hopf solution

$$
f_{1}(x)=f_{1 / 2}\left[x-\frac{1}{2} f_{1}(x)\right]
$$

with $f_{1 / 2}(x)=i \pi \rho_{1 / 2}(x)$ and

$$
f_{1}(x)=x-\frac{1}{2} U^{\prime}(x)+i \pi \rho(x)
$$

To determine $f_{1}$ we replace $x \rightarrow x^{\prime}=x-f_{1}(x) / 2$ and $r(x) \rightarrow r\left(x^{\prime}\right)=\pi \rho_{1 / 2}\left(x^{\prime}\right) / 2$ in equation (5.2). As a consequence of (5.3)

$$
r\left(x^{\prime}\right)=-\frac{i}{2} f_{1}(x)
$$

which we can substitute into (5.2) to get a quadratic equation on $f_{1}$

$$
\left(1-k x^{2}\right)\left(x-f_{1}\right)^{2}-m^{2} x\left(x-f_{1}\right)-b+x^{2}=0 .
$$

Solving this quadratic equation we obtain the final value of the function $f$

$$
f_{1}(x)=x-\frac{m^{2} x}{2\left(1-k x^{2}\right)}+\frac{i}{1-k x^{2}} \sqrt{\frac{m^{4} x^{2}}{4}+\left(b-x^{2}\right)\left(1-k x^{2}\right)}
$$


and, therefore

$$
\left\{\begin{array}{l}
U^{\prime}(x)=\frac{m^{2} x}{1-k x^{2}} \\
\pi \rho(x)=\frac{1}{1-k x^{2}} \sqrt{\frac{m^{4} x^{2}}{4}+\left(b-x^{2}\right)\left(1-k x^{2}\right)} .
\end{array}\right.
$$

The original definition of the matrix chain deals, however, with a rescaled potential $V(M)$ related to $U(x)$ by (2.9). In our model

$$
V^{\prime}(x)=\frac{\kappa^{2} x+G x^{3}}{1-G \epsilon^{2} x^{2} / 2}
$$

where we introduced the "scaled" mass $\kappa$ and the coupling constant $G$

$$
\left\{\begin{array}{c}
m^{2}=2+\kappa^{2} \epsilon^{2} \\
k=\frac{1}{2} G \epsilon^{3} .
\end{array}\right.
$$

As always, by rescaling all matrices in the matrix chain partition function (1.1) we can make $\kappa^{2}=V^{\prime \prime}(0)$ anything we want. Changing the value of $\kappa^{2}$ simply changes the scale of $\epsilon$ and shifts the position of all critical points. We shall adhere to the convention of (2.31) that was used for pure quartic potentials and set $\kappa^{2}=-4$. In terms of the original parameters $m^{2}$ and $k$ this would mean

$$
\left\{\begin{aligned}
m^{2} & =-2\left(2 \epsilon^{2}-1\right) \\
k & =\frac{1}{2} G \epsilon^{3},
\end{aligned}\right.
$$

a relation quite similar to (2.32).

The resulting potential $V(x)$ is plotted in fig.4. It goes to infinity as $x \rightarrow \pm x_{\max }=$ $\sqrt{2 / \epsilon^{2} G}$ thereby restricting the eigenvalues to a finite range $\left[-x_{\max }, x_{\max }\right]$. Note that the plot of fig. 4 makes sense only when $\kappa^{2} x_{\max }+G x_{\max }^{3}>0$-otherwise for $x \rightarrow \pm x_{\max }$ the potential goes to minus, not plus infinity and therefore is unbounded from below. For $\kappa^{2}=-4$ this constraint translates into $\epsilon<1 / \sqrt{2}$ or, equivalently, $m^{2}>0$.

Such a picture is certainly consistent with what we already know. Indeed, at $\epsilon=1 / \sqrt{2}$ we expect $\rho(x)$ to develop a logarithmic singularity. On the other hand, the solution (5.7) cannot, by construction, have such logarithms. Therefore, it is perfectly natural that this solution should degenerate precisely at $\epsilon=1 / \sqrt{2}$. Furthermore, our exact solution has no singularities for $\epsilon$ between 0 and $1 / \sqrt{2}$. Here is an example of a special situation when the terms responsible for such singularities - say, the terms in the curly brackets in (3.25) are arranged to vanish. In fact, the exact eigenvalue density (5.7) can be used to verify and confirm all of the perturbative techniques developed in section 3 . For instance, when $b=0$ 

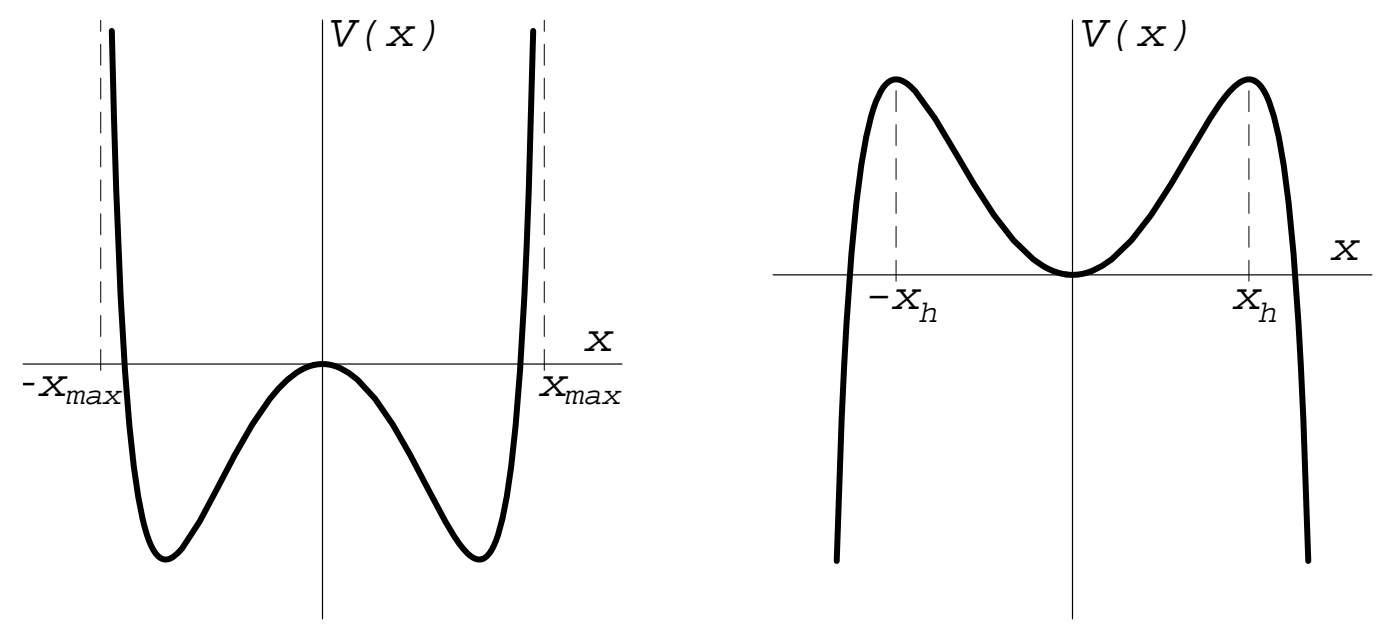

Fig. 4: An exactly solvable Penner-type potential $V(x)$ for (right) $\kappa^{2}<0$ and $G>0$ or (left) $\kappa^{2}>0$ and $G<0$.

adapting the small $x$ asymptotic series of (3.19) to the case of our nonpolynomial potential yields precisely the expansion of the exact solution (5.7) in powers of $x$. Moreover, for $b \neq 0$ it is possible to calculate, up to arbitrarily high orders, the small $b$ expansion similar to (3.30). The result, not surprisingly, can be summed into an exact formula thereby providing a nontrivial check of our computational methods.

Quite remarkably, the same exact solution (5.7) can be used to rediscover not only the singularity at $\epsilon=1 / \sqrt{2}$ but also the Kosterlitz-Thouless phase transition. To see this consider the same potential (5.8) but with $\kappa^{2}>0$ and $G<0$. Such a potential, also sketched in fig. 4, has two humps at $x_{\mathrm{h}}= \pm \kappa / \sqrt{|G|}$ which play exactly the same role as the single hump at $x=0$. By analogy with (2.31) we shall fix the second derivative of the potential at the hump to be $V^{\prime \prime}\left(x_{\mathrm{h}}\right)=-4$. This imposes the following restriction on $\kappa$

$$
\kappa^{2}=\frac{2}{1-\epsilon^{2}}
$$

That is, the potential degenerates as we approach the Kosterlitz-Thouless phase transition at $\epsilon=1$. At that point expression (5.7) cannot possibly give the correct eigenvalue density. Such "nontranscendental" form of $\rho$ does not contain the requisite logarithmic singularity which, as we found, has to be present for any, even nonpolynomial, interaction potential.

Most likely, one could use the same methods to construct the exact solutions that would describe the logarithmic singularities themselves. The idea would be to invent a 
globally defined $\rho_{1 / 2}(x)$ having both the small $x$ and small $b$ expansions consistent, to first order, with (3.23), (3.44) and (4.24). Then evolving it to $t=1$ would, by construction, generate the $\rho(x)$ with an adequate amount of logarithms. After the global $\rho(x)$ has been found the direct computation of the free energy and various critical indices becomes reasonably straightforward. It would certainly be most interesting to actually carry out this program, especially for the Kosterlitz-Thouless phase transition where the results would have a direct meaning in terms of string theory.

\section{Interaction of Vortices with Curvature Defects}

We have seen throughout this paper that the Kosterlitz-Thouless phase transition and the subleading logarithmic singularities of the $\epsilon<1$ phase bear remarkable mathematical similarity. This similarity is perhaps expressed best by the formulas for $\rho_{1 / 2}(x)$. Indeed, an explicit expression for $\rho_{1 / 2}$ at $\epsilon=1 / \sqrt{2}$,

$$
\pi \rho_{1 / 2}(x)=\sqrt{2 b+4 x^{2}+g u(x)}
$$

with

$$
\begin{aligned}
u(x)= & \frac{8}{\pi} x\left(2 x^{2}+\frac{b}{2}\right) \sqrt{x^{2}+\frac{b}{2}} \log \left[\frac{\sqrt{x^{2}+b / 2}+x}{\sqrt{x^{2}+b / 2}-x}\right] \\
& -2 r_{2}\left(2 x^{2}+\frac{b}{2}\right)^{2}-b\left(2 x^{2}+\frac{b}{2}\right)
\end{aligned}
$$

has precisely the same structure as its analogue for $\epsilon=1$, equation (4.24).

Such parallels suggest that the qualitative physical picture of logarithmic singularities must be related to the vortex picture of the Kosterlitz-Thouless transition. Below we shall argue that this is indeed the case.

In continuum theory, the action for a one-dimensional noncritical string compactified on a circle of radius $R$ is

$$
S[g, X]=\frac{1}{\pi} \int d^{2} x \sqrt{g} g^{\alpha \beta} \partial_{\alpha} X \partial_{\beta} X
$$

where $X$ is a compactified string coordinate, $x \equiv X+2 \pi n R$ for any $n \in \mathbb{Z}$ and $g_{\alpha \beta}-$ the two-dimensional worldsheet metric. For flat $g_{\alpha \beta}$ a typical vortex-a solution of the equations of motion with a nonzero winding number $n$-is given by

$$
X(r, \phi)=n \phi R
$$


$r$ and $\phi$ being the polar coordinates parametrizing the flat area of the worldsheet. The value of the action for such a vortex would be infinite were it not for the cutoffs. Let us therefore introduce the infrared cutoff $L$ and the ultraviolet cutoff $a$ (say, the lattice spacing if the worldsheet is discretized.) With these cutoffs the vortex action equals

$$
S_{\text {vort }}=2 n^{2} R^{2} \log \left(\frac{L}{a}\right) .
$$

The total weight with which a vortex contributes to the partition function of the whole theory can be found by multiplying the number of places where the vortex could be centered (which is simply the total number of lattice sites $N_{\text {tot }} \sim(L / a)^{2}$ ) by the Gibbs factor $\exp \left(-S_{\text {vort }}\right)$

$$
w_{n} \propto\left(\frac{L}{a}\right)^{2} \mathrm{e}^{-S_{\mathrm{vort}}}=\left(\frac{L}{a}\right)^{2\left(1-n^{2} R^{2}\right)} .
$$

As a result, depending on the value of $R$ vortices may or may not be important [10]. If $R>1$ one has $2\left(1-n^{2} R^{2}\right)<0$ for all integer $n$ and the total weight of any vortex vanishes when the cutoffs are removed. In this case vortices can be neglected. On the contrary, for $R<1$ the weight $w_{1}$ goes to infinity whenever $L / a \rightarrow \infty$ and so vortices do matter. The two phases which arise are separated by a Kosterlitz-Thouless phase transition with the critical value of compactification radius $R_{\mathrm{cr}}=1$.

Let us now imagine that the two-dimensional worldsheet is not completely flat but can have freely moving conical singularities. Around a conical singularity such a worldsheet can still be described by the polar coordinates $r$ and $\phi$ but with $\phi$ running in a different interval $0 \leq \phi \leq 2 \pi+\alpha$. The "excess angle" $\alpha$ characterizes the amount of curvature acquired by the manifold due to the conical singularity.

For a vortex centered right at the top of the cone the vortex field $X$ would be given by

$$
X(r, \phi)=\frac{2 \pi}{2 \pi+\alpha} n \phi R
$$

Consequently, the vortex action changes as well,

$$
S_{\text {cone }}=\frac{4 \pi}{2 \pi+\alpha} n^{2} R^{2} \log \left(\frac{L}{a}\right)
$$

As we see, if $\alpha>0$ - that is, when the conical singularity contributes negative curvaturethe value of the vortex action decreases compared to flat space. Furthermore, in twodimensional gravity it does not cost any extra energy to create a conical singularity, so long as the total worldsheet area and the genus remain the same. Therefore, we could contemplate a Kosterlitz-Thouless phase transition induced by such pairs of "correlated" 
cones and vortices centered closely to each other. The critical value of $R$ or the corresponding $\epsilon=1 / R$ for this transition can be found similarly to (6.6),

$$
\epsilon_{\mathrm{cr}}(\alpha)=\sqrt{\frac{2 \pi}{2 \pi+\alpha}}<1 .
$$

Of course, the pairs of cones and vortices with nearby centers form only a small susbset of all possible states in our system 5 . Roughly, the numbers of possible positions of either a vortex or a conical tip are $(L / a)^{2}$ each, adding up to a total of $\sim(L / a)^{4}$ states. Only $\sim(L / a)^{2}$ of them are the states where the cone and the vortex have the same center. Consequently, the contribution of such "correlated pairs" can produce at most a finite size correction to the free energy which is down by $(a / L)^{2} \propto 1 / A$ for a worldsheet of area $A$. But this is exactly what we find from (3.23). There the logarithmic correction to $\rho(x)$ is also subleading. Indeed, at $\epsilon=1 / \sqrt{2}$ the leading contribution to the free energy - the one which is important in the double scaling limit - is associated with the first term of expansion for the eigenvalue density, $\rho(x) \propto|x|+\ldots$. For any $\epsilon<1$ this contribution is simply the standard $c=1$ free energy,

$$
\mathcal{F}(\delta g) \propto \frac{\delta g^{2}}{|\log \delta g|}+\mathcal{O}\left(\delta g^{3}\right)
$$

The logarithmic correction $x^{3} \log [1 /(\lambda x)]$ would modify only the $\mathcal{O}\left(\delta g^{3}\right)$ terms of $(6.10)$. And, as it turns out, such $\mathcal{O}\left(\delta g^{3}\right)$ terms correspond precisely to $1 / A$ corrections.

To see this, we just have to recall that the cosmological constant $\delta g$ and the worldsheet area $A$ are a pair of thermodynamically conjugate variables. That is, the string theory partition function with a nonzero $\delta g$, given by $\mathcal{F}(\delta g)$, is related to the partition function of random surfaces with fixed total area $\mathcal{Z}_{\text {str }}(A)$ by the formula

$$
\mathcal{Z}_{\text {str }}(\delta g) \equiv \mathcal{F}(\delta g)=\int_{0}^{\infty} d A \mathrm{e}^{-\delta g A} \mathcal{Z}_{\text {str }}(A)
$$

Consequently, an expansion in powers of $\delta g$ arising in (6.10) would translate into a $1 / A$ expansion for the fixed area partition function and the fixed area free energy.

5 Here is, perhaps, the most objectionable part of this argument. Strictly speaking, the Kosterlitz-Thouless picture can be used reliably to infer the existence of a phase transition only for the whole system, and not for an artificially chosen small subset of states. The only justification for what we say is that the results gotten via such reasoning do not contradict the exact computations of sections 3 and 4 . 
Finally, this picture can be used to explain why the logarithmic singularities form a discrete set. The reason is, for a discretized surface generated via a matrix model the excess angle $\alpha$ at any graph vertex can assume only certain discrete values. For example, a matrix model with a cubic potential would produce surfaces composed of equilateral triangles. On such surface the angle $\alpha$ corresponding to a vertex with the coordination number $q$ (where $q$ triangles meet together) equals

$$
\alpha=\frac{\pi}{3}(q-6) \text {. }
$$

We see that $\alpha$ and therefore the critical lattice spacings computed from (6.9) do come out discrete. However, this rough estimate fails to yield the exact critical values of $\epsilon$. This should not be a surprise. To get these critical values right one would have to take into account various fluctuations that we neglected. For instance, the picture of an almost flat lattice with just a few curvature defects scattered around is certainly quite inaccurate. In reality, almost every vertex of a generic two-dimensional graph would have $q \neq 6$. It would be quite interesting to find out whether such effects can be taken into account and whether our rough picture survives that.

To summarize, the logarithmic singularities at $\epsilon<1$ carry along lattice information. That is, their position and order depend on the particular type of polygons tiling our random surface. However, this does not yet mean that such effects are of little interest. For example, the Kazakov's multicritical points in one-matrix models [22] provide, also through a very special way of tiling, a description of minimal models coupled to quantum gravity. There the connection with continuum theory is not obvious from the matrix model formulation. A similar indirect continuum interpretation might exist for the logarithmic singularities in the matrix chain. In any case, the subleading singularities of the $\epsilon<1$ phase are quite an unusual phenomenon for matrix models. Indeed, in most theories the whole singularity structure is captured by the double scaling limit of the matrix model, the corrections to this limit being perfectly regular. For the matrix chain this does not seem to be so, which perhaps is rather satisfactory. As an example, it is very well known [7] that the double scaling limit of the chain model is smooth throughout the interval $0 \leq \epsilon<1$ and describes $c=1$ string theory. This leads to a surprising conclusion that the discrete structure of the target space has no effect at all. We see that although such a conclusion is absolutely true in the continuum theory, the target space discreteness does show through a nontrivial set of corrections.

Finally, the logarithmic singularities can be of interest for a separate reason. It appears remarkable that such a complicated family of singularities arises precisely on the boundary between the $c<1$ and $c>1$ theories. One might wonder, maybe somewhat groundlessly, whether a similar kind of structure could also emerge in any of the possible $c>1$ models. 


\section{Conclusions}

We have investigated, in the large $N$ limit, the phase structure and the eigenvalue density of the one-dimensional infinite random matrix chain. We developed a systematic expansion applicable in the vicinity of the Kosterlitz-Thouless phase transition as well as throughout the $\epsilon<1$ phase of the model. Remarkably, such expansion can be used to extract the critical properties of the eigenvalue density even when the model is hard to solve exactly.

It turned out that the infinite matrix chain exhibits a few effects rather unusual in matrix models. In particular, we found an infinite number of subleading (not important in the double scaling limit) critical points, similar in nature to the Kosterlitz-Thouless phase transition. These special points appear to arise from interactions of vortices with curvature defects and depend on the specific type of polygons tiling a random surface.

The computational tools that were required here, such as the hydrodynamic representation and the functional equation $G_{+}\left[G_{-}(x)\right]=x$ are likely to have many more applications. Even in the case of the matrix chain these methods reproduce and generalize with great ease the results which would look nontrivial from other viewpoints. They can also be used to explore the $\epsilon>1$ phase and to establish the parallels with the character expansions.

In addition, there are a number of specific open problems that should be possible to resolve with our tools. One such problem is to compute carefully the free energy of the matrix chain at the Kosterlitz-Thouless point. This could be done via the procedure outlined at the end of section 5. Furthermore, it must be possible to relate the critical behavior of the eigenvalue density $\rho(x)$ that we now know, to the physical picture of vortex interactions. Finally, it would be very interesting to evaluate, or at least to represent in terms of the Hopf equation, the correlation functions of the matrix chain. Such representation would help to separate and compute the contributions of vortices with different vortex charges. As a consequence of this computation, we shall be able to study in a very direct fashion the dynamics of vortices interacting with quantum gravity.

It is our pleasure to thank D. Gross, V. Kazakov and A.A. Migdal for their interest, support and for helpful advice. We are especially grateful to J. Goldstone for a discussion concerning equation (3.38). Finally, we are indebted to E. Farhi and U.-J. Wiese for ideas regarding the numerical aspects of this problem.

This work is supported in part by funds provided by the U.S. Department of Energy under cooperative research agreement DE-FC02-94ER40818 and the Swiss National Science Foundation. 


\section{References}

[1] D. Gross and A. Migdal, Nucl. Phys. B340 (1990) 333;

E. Brézin and V. Kazakov, Phys. Lett. B236 (1990) 144;

M. Douglas and S. Shenker, Nucl. Phys. B335 (1990) 635.

[2] D. Gross and N. Miljković, Phys. Lett. B238 (1990) 217;

E. Brézin, V. Kazakov and A. Zamolodchikov, Nucl. Phys. B338 (1990) 673.

[3] D. Gross, The $c=1$ Matrix Models, in: Two Dimensional Quantum Gravity and Random Surfaces (World Scientific, Singapore, 1992) p.143

[4] F. David, A Scenario for the $c>1$ Barrier in Noncritical Bosonic Strings, report SACLAY-SPHT-96-112, October 1996, hep-th/9610037.

[5] T. Eguchi and H. Kawai, Phys. Rev. Lett. 48 (1982) 1063;

A. Migdal, Phys. Rep. 102 (1983) 199.

[6] G. Parisi, Phys. Lett. B238 (1990) 213.

[7] D. Gross and I. Klebanov, Nucl. Phys. B344 (1990) 475;

Nucl. Phys. B354 (1991) 459.

[8] I. Kostov, Nucl. Phys. B376 (1992) 539.

[9] D. Boulatov and V. Kazakov, Int. J. Mod. Phys. A8 (1993) 809;

Nucl. Phys. Proc. Suppl. 25A (1992) 38.

[10] V. Berezinskii, JETP 34 (1972)610;

J.M. Kosterlitz and D. Thouless, J. Phys. C6 (1973) 1181;

J. Villain, J. Phys. C36 (1975) 581.

[11] G. Parisi, Phys. Lett. B238 (1990) 209; Europhys. Lett. 11 (1990) 595;

G. Parisi, G. Salina and A. Vladikas, Phys. Lett. B256 (1991) 397.

[12] D. Gross and E. Witten, Phys. Rev. D21 (1980) 446.

[13] M. Douglas and V. Kazakov, Phys. Lett. B319 (1993) 219.

[14] E. Brézin, C. Itzykson, G. Parisi and J.B. Zuber, Commun. Math. Phys. 59 (1978) 35.

[15] C. Itzykson and J.B. Zuber, J. Math. Phys. 21 (1980) 411.

[16] A. Matytsin, Nucl. Phys. B411 (1994) 805.

[17] D. Boulatov, Mod. Phys. Lett. A9 (1994) 1963;

A. Matytsin and A. Migdal, Int. J. Mod. Phys. A10 (1995) 421;

J.-M. Daul, Q-states Potts Model on a Random Planar Lattice, ENS report, November 1994, hep-th/9502014.

[18] V. Kazakov, M. Staudacher and T. Wynter, Commun. Math. Phys. 177 (1996) 451;

Nucl. Phys. B471 (1996) 309. 
[19] D. Gross and A. Matytsin, Nucl. Phys. B429 (1994) 50; Nucl. Phys. B437 (1995) 541;

J. Minahan and A. Polychronakos, Nucl. Phys. B422 (1994) 172.

[20] Yu. Makeenko, Phys. Lett. B314 (1993) 197;

Int. J. Mod. Phys. A10 (1995) 2615;

L. Paniak and N. Weiss, J. Math. Phys. 36 (1995) 2512.

[21] D. Gross, Phys.Lett. B293 (1992) 181.

[22] V. Kazakov, Mod. Phys. Lett. A4 (1989) 2125. 